\title{
The Role of the Dipole Interaction of Molecules with Charged Particles in the Polar Stratosphere
}

\author{
Yury Belikov and Sergey Nikolayshvili \\ Fedorov Institute of Applied Geophysics, 9 Rostokinskaya Str., Moscow 129128, Russia
}

\begin{abstract}
Our analysis of published results of experiments in the Polar Regions substantiates and further develops our new approach to the photochemical processes in the polar stratosphere involving the charged particles. The dipole interaction of molecules with charged particles, primarily with ions, leads to the adhesion and disintegration of a number of molecules including ozone. Molecules acquire additional energy on the surface of the charged particles, enabling reactions that are not possible in space. Galactic cosmic rays are the main source of ions in the polar stratosphere, their equilibrium concentration at altitudes of 15 to $25 \mathrm{~km}$ can reach up $(1-5) \times 10^{3}$ ions $/ \mathrm{cm}^{3}$. Estimations show that if the ozone destruction in the regime of "collision" with ions then the lifetime of ozone will vary from 10 days to 2 months. We suppose that alongside with the chlorine mechanism of ozone destruction there is a mechanism of ozone decay on a charged particle which can act also at those latitudes and altitudes where chlorine oxide ClO is absent, as well as in the night conditions. Here, we demonstrated the close connection of photochemical processes with the dynamic, electrical and condensational phenomena in the stratosphere, in particular, with the accumulation of unipolar charged particles on the upper and lower boundaries of the polar stratospheric clouds and aerosol layers as a result of the activity of the global electric circuit.
\end{abstract}

Key words: Polar stratosphere, ozone, charged particles, dipole interaction, global electric circuit, photochemistry.

\section{Introduction}

The Earth's atmosphere, in particular the polar stratosphere, contains a large number of charged particles of various origins. This includes charged water droplets, ice particles and ice crystals, as well as charged aerosols of various origins, positive and negative ions, condensation nuclei and others. The size of charged particles varies from a few nanometres to hundreds of microns.

Galactic cosmic rays are the main source of the charge in the polar stratosphere and are responsible for the formation of large reservoir of ions. The rate of primary ion generation in the lower stratosphere is about of $\sim 10$ pairs $/\left(\mathrm{cm}^{3} \cdot \mathrm{s}\right)[1,2]$. The equilibrium ion concentration (of each sign) at the altitude range of $\sim 15-25 \mathrm{~km}$ may reach values of $\sim(1-5) \times 10^{3}$ ions $/ \mathrm{cm}^{3}$ $[2,3]$.

The importance of the role of the dipole interaction

Corresponding author: Yury Belikov, doctor of sciences, head of laboratory, research fields: physics and chemistry of atmosphere. E-mail: yury_belikov@mail.ru. of molecules with charged particles in the atmosphere in condensation and droplet formation has been demonstrated in Refs. [4, 5] and in other works. The significance of the ion-molecule reactions in the atmospheric photochemistry and the connection of these reactions with the ozone have been investigated by a number of researchers.

For example, the existence of an ion catalytic cycle involving ozone was suggested in Refs. [6, 7]. Morita and Ishikawa [3] theorize that variations in the ionic composition and the concentration of ozone in the stratosphere are associated with the adhesion of ions to ozone molecules.

There are a large number of the experimental investigations on the interaction of ions with molecules [8-12]. For example, the reaction between $\mathrm{HBr}$ and the negative ion $\mathrm{OH}^{-}\left(\mathrm{H}_{2} \mathrm{O}\right)_{\mathrm{n}}$ for $0 \leq \mathrm{n} \leq 11$ at $100 \mathrm{~K}$ has been studied in Ref. [8]. This reaction yields product ions in the form of $\mathrm{Br}^{-}\left(\mathrm{H}_{2} \mathrm{O}\right)_{\mathrm{m}}$, which differ only in the degree of solvation of the core ion. In our opinion, a similar reaction of an $\mathrm{HCl}$ molecule 
with a positive ion in the form of $\left(\mathrm{H}_{3} \mathrm{O}^{+}\right)\left(\mathrm{H}_{2} \mathrm{O}\right)_{n} \mathrm{OH}$ can result in the formation of the additional water molecule and the active $\mathrm{Cl}$ atom. In this study, we suggest that positive ions with a core $\mathrm{H}_{3} \mathrm{O}^{+}$can play a key role in the stratospheric photochemistry.

There are great numbers of works on the cosmic rays and charged particles effect on the photochemical and other processes in the stratosphere.

Varotsos et al. [13] found a strong correlation between the muon flux from the underground detector and the effective temperature of the stratosphere, which suggests a possible connection between cosmic rays and the ozone content via a change in the stratospheric dynamics as a result of temperature changes.

In Ref. [14] Yu pointed out the possibility of the large NAT particles formation and also the denitrification of the stratosphere as a result of the cosmic rays and solar activity effects. He proposes that high energy comic ray particles might induce the freezing of the supercooled $\mathrm{HNO}_{3}-\mathrm{H}_{2} \mathrm{O}-\mathrm{H}_{2} \mathrm{SO}_{4}$ droplets when they hit and penetrate these droplets in the polar stratosphere.

$\mathrm{Lu}$ and Sache [15] proposed the impact of cosmic rays on particles of PSCs (polar stratospheric clouds) with the formation of active chlorine. Their results demonstrated that a strong correlation between ozone depletion and the intensity of galactic cosmic rays exists during in the polar spring. According to Ref. [15] and other work of $\mathrm{Lu}$ et al. [16-18], the secondary electrons arising from the molecules ionization by the cosmic rays are absorbed by the solid ice particles of the polar stratospheric clouds. There, on those particles surfaces, the specific reactions of the chlorine compounds with electrons take place which release the active chlorine. The example of such reaction [18] is given below:

$\mathrm{e}_{\mathrm{t}}^{-}\left(\mathrm{H}_{2} \mathrm{O} / \mathrm{NH}_{3}\right)_{\mathrm{n}}+\mathrm{CF}_{2} \mathrm{Cl}_{2} \rightarrow \mathrm{CF}_{2} \mathrm{Cl}_{2} *^{-} \rightarrow \mathrm{Cl}^{-}+\mathrm{CF}_{2} \mathrm{Cl}$, where $\mathrm{e}_{t}^{-}$is a weakly-bound electron trapped in the polar $\left(\mathrm{H}_{2} \mathrm{O} / \mathrm{NH}_{3}\right)$ ice. The chlorine ion, generated in this reaction loses the charge in the process of collision with positive ions, or with its surrounding molecules. Further on, the chlorine atom acts in the well-known catalytic cycles, destructing the ozone molecules. Based on this mechanism, which is called "cosmic-ray-driven electron-induced reaction mechanism (or theory)", Lu et al. developed a climatic change theory, where the main role is played not by $\mathrm{CO}_{2}$, but by CFCs and cosmic rays [17-20].

In Ref. [21] we proposed and provided evidence for the ozone destruction mechanism on ice crystals charged by ions in the polar stratosphere. We suggested there that the ozone and various compounds destruction took place not at the total surface of the crystal but on the sharp juts of ice crystals [21]. Under the strong electric field condition the polar molecules accumulate at those juts, some of these molecules can destruct, and non-polar molecules destruction is also possible (see below). Our mechanism proposed not only the traditional mechanism of the ozone depletion, but the direct ozone destruction also.

The further investigations are necessary for clarification of the questions about contribution of the charged ice particles into the ozone destruction; what charge do they bear, either ionic, electronic, or mixed one; and what is the mechanism of the ozone destruction.

In Ref. [22] we proposed and here, in this work, we develop an idea that our earlier proposed mechanism can be implemented not only on the ice crystal surface, but also in the free atmosphere on the effective ion surface.

Our mechanism allows to explain the ozone destruction in the nighttimes' conditions and at those heights and latitudes where chlorine monoxide $\mathrm{ClO}$ either absent, or its concentration is low. In the work presented here we make an addition to that mechanism and show the possibility of the direct depletion of ozone and a set of other compounds, including halogen-containing molecules on the positively charged particles of the nanometer size range (mainly ions). In our opinion, just such particles accumulate 
on the upper boundary of the polar stratospheric clouds as a result of the global electric circuit work.

We proposed previously that some photochemical reactions in the polar stratosphere can pass in a conjunction with charged particles [21, 22]. How can charged particles affect the photochemical processes in the atmosphere? First of all, those processes can be triggered by the acceleration of the chemical reactions which involve charged particles. In this case, a molecule with a dipole moment acquires additional energy on the surface of a charged particle. This energy can be used in chemical reactions at the surface. Reactions on the surface of highly charged particles are particularly effective. We proposed that this is analogous to the charged sharp juts of ice crystals [21]. In this paper, we argue for the idea that individual ions can also act as highly charged or supercharge particles. Furthermore, we previously emphasized that a charged particle is like a type of filter, forming its own environment of molecules with high dipole moments $[21,22]$. The explanation of this phenomenon is following:

Some complex molecules can break up on the surface of a charged particle into simpler molecules. The lifetime of those simple molecules on the surface of the particle strongly depends on their dipole moment. Molecules with a high dipole moment remain on the surface and may participate in further reactions there, whereas molecules with a low dipole moment are quickly ejected from the surface of the charged particles into space.

For example, $\mathrm{HOONO}$ (the isomer of $\mathrm{HNO}_{3}$ ) can decompose to $\mathrm{OH}$ and $\mathrm{NO}_{2}$ on the surface of the supercharged particle [21], and then $\mathrm{NO}_{2}$ molecules which have a small dipole moment are ejected into space while hydroxyl radical $\mathrm{OH}$ remains on the charged particle surface. Further on hydroxyl radical can react with various compounds molecules, which exist in space and attach to the charged particle surface. This fact allows to explain the destruction in the polar stratosphere of such stable molecules as $\mathrm{HCl}$ and $\mathrm{N}_{2} \mathrm{O}$, and also non-polar molecules like $\mathrm{CH}_{4}, \mathrm{CCl}_{4}$. In fact the destruction of some molecules takes place not directly but with the help of intermediary agent, i.e. $\mathrm{OH}$. Not only HOONO can be the source of hydroxyl radical on the charged particle surface, but some other compounds. This question will be considered later.

In this study, we develop and support our hypothesis based on the analysis of the research findings from the published experiments in the Polar Regions. The important result of our analysis is that ozone molecules can be destroyed by interacting with charged particles. The charged particles present not only in the stratosphere, but also in the troposphere and in the mesosphere, as well as in the surface boundary layer. Therefore, it is necessary to take into account their effect to interpret the numerous data which were accumulated by ground-based stations [23, 24].

\section{Dipole Interaction and Chemical Reactions on the Surface of Charged Particles}

A force which appears between a molecule with a dipole moment $p$ and a charged particle with a charge $\mathrm{q}$ as a result of the dipole interaction that for a sufficiently large distance $r$ between the particle and the molecule, can be estimated as Ref. [25]:

$$
\mathrm{F}=\mathrm{pdE} / \mathrm{dr}=|\mathrm{pq}| /\left(2 \pi \varepsilon_{0} \mathrm{r}^{3}\right)
$$

Here, $\mathrm{E}$ is the electric field of the particle at a distance $r$, and $\varepsilon_{0}$ is the electric constant. In the Eq. (1), it is assumed that the dipole is oriented along the electric field. The effect of this force or a random collision could cause a molecule to appear on the surface of a charged particle. After the molecule encounters the surface of the charged particle, the energy of the particle increases. The energy possessed by a molecule on the surface of a spherical charged particle with radius $r_{0}$ can be expressed as Ref. [25]:

$$
\mathrm{W}_{0}=-|\mathrm{pq}| /\left(4 \pi \varepsilon_{0} \mathrm{r}_{0}^{2}\right)
$$

Fig. 1 shows two molecules, $M_{1}$ and $M_{2}$. At the chemical reaction of these molecules on the surface of a charged particle the additional energy equal to the 


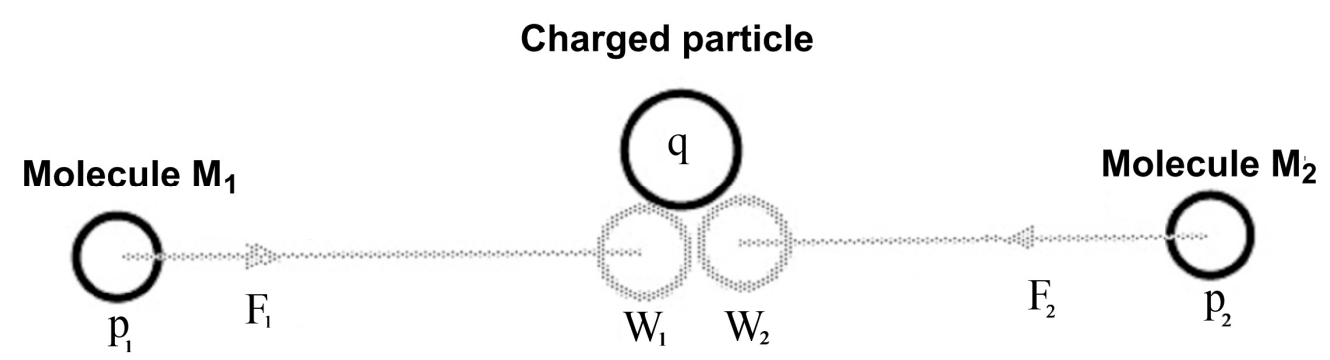

Fig. 1 Dipole interaction of molecules $M_{1}$ and $M_{2}$, with dipole moments $p_{1}$ and $p_{2}$, respectively, with a charged particle of charge $q$. The forces acting on the molecules $M_{1}$ and $M_{2}$ are shown, as well as the respective energies $W_{1}$ and $W_{2}$ acquired by molecules on the surface of the charged particle. These energies can be used by molecules during chemical reactions on the particle surface.

sum of the energies of the two molecules $\mathrm{W}_{1}+\mathrm{W}_{2}$ may be utilized. Examples of dissociation reactions in which a water molecule is one of the molecules are given below.

The above given formula shows that the energy acquired by the molecules depends on the ratio of the particle charge to the square of its radius. If this ratio is small, then the increase in the reaction rate at the surface compared with the reaction rate in the volume will be also low.

For highly or maximally charged spherical particles, the following relationship holds in the SI system between the ionic charge and the square of the radius of the solid particle [26]:

$$
\mathrm{Q}_{\max }=2.22 \mathrm{r}^{2} \text {. }
$$

In the Eq. (3), we have recalculated the coefficient from CGSE unities given in Ref. [26] into SI units. This relation is obtained theoretically for maximal ionic charge [26]. For droplets of water, there is also a limiting charge at which the drop breaks because of the close vicinity of unipolar charges in the case of excess charge (Rayleigh limit, see also Fig. 2) [26].

Substituting Eq. (3) into Eq. (2), we find that:

$$
\mathrm{W}_{\max }=-0.415|\mathrm{p}|
$$

where the units of $\mathrm{W}_{\max }$ are $\mathrm{eV}$ and those of $|\mathrm{p}|$ are Debye units.

We define supercharged particles as highly charged particles or particles which properties are close to these particles. It is these particles that in our opinion play a key role in the stratospheric photochemistry. A feature of the supercharged particles is that the reactions on their surface can overcome an energy barrier of $\sim 1 \mathrm{eV}$, and a variety of compounds such as $\mathrm{ClOOCl}, \mathrm{ClONO}_{2}, \mathrm{O}_{3}, \mathrm{HOONO}, \mathrm{HO}_{2} \mathrm{NO}_{2}, \mathrm{~N}_{2} \mathrm{O}_{5}$ may be dissociated on the surface of such particles (see Table 1 below). However, where in the stratosphere do such highly charged particles arise? We hypothesized that the sharp juts of a charged ice crystals could act as such particles [15]. These juts of small size can serve as sinks of charge, which accumulate in the large crystal, representing the maximal charge of these juts. In the present study we argue that individual ions can also act as the supercharged particles.

Fig. 2 shows the relationship between particle size and the possible maximum charge. In the figure, this relationship is shown both for solid particles (charge $\mathrm{Q}_{\text {solid}}$ ) and for drops (charge $\mathrm{Q}_{\text {drop }}$ ) [26, 27]. It also shows the average charge $Q_{\text {avr }}$ for particles of different sizes in a cloud in the troposphere [27].

From this figure, it is clear that for large particles to become supercharged, such a large charge is necessary that in our opinion, it is unlikely to be present under stratospheric conditions. Especially large, highly charged drops of water have a much lower charge than maximally charged solid particles of the same size, so a drop of water is not, by virtue of its physicochemical properties, a suitable supercharged particle.

The analysis of Fig. 2 shows that very small particles with a small charge have properties that are close to those of the supercharged particles. Fig. 2 shows a straight line corresponding to the elementary 


\section{Radius of the particle, $\mathrm{m}$}

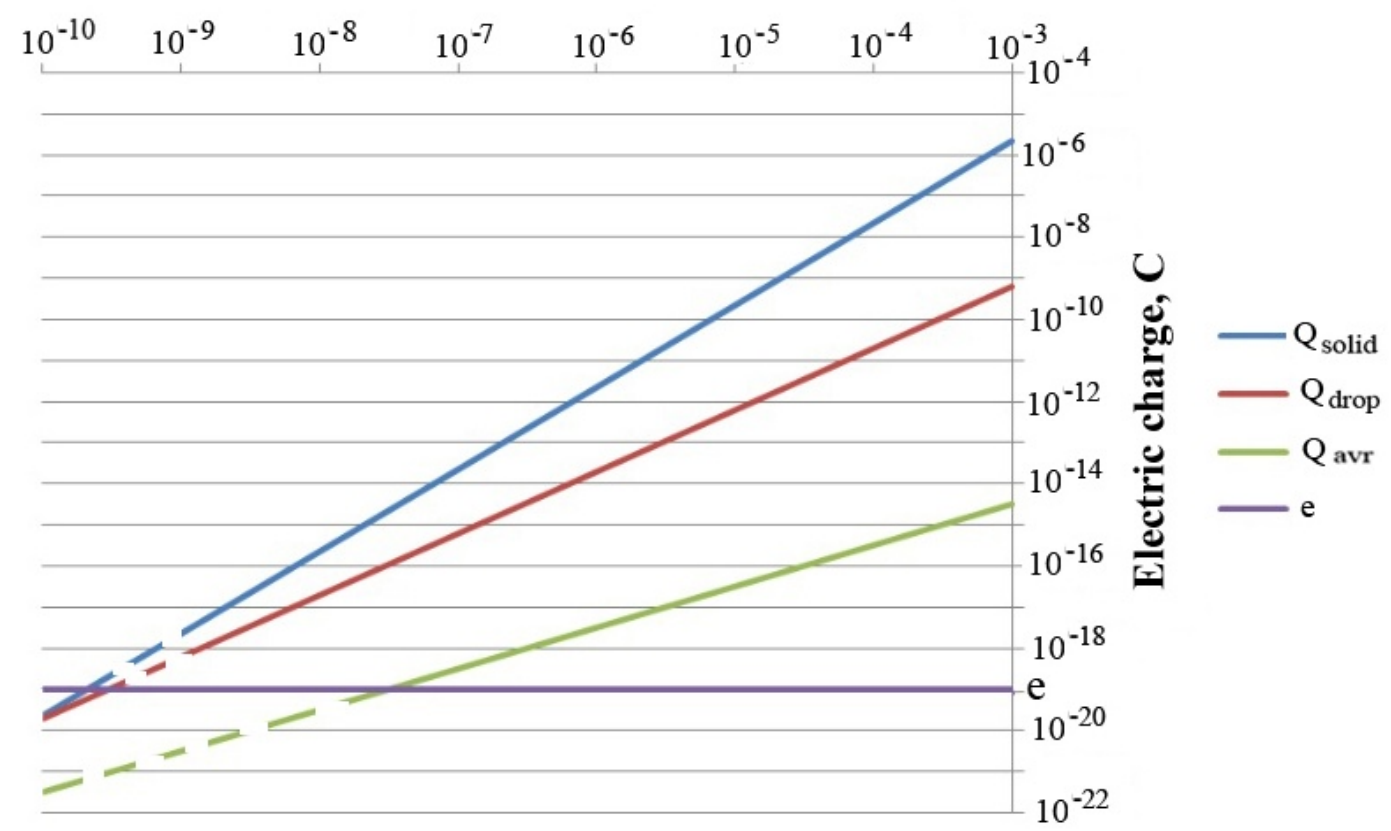

Fig. 2 The ratio between ionic charge and particle size for highly charged solid particles (blue line) and maximally charged water droplets (red line) [26, 27] are given. The average charge of cloud particles in the troposphere [27] is also shown (green line). The relationship between ionic charge and particle size is extrapolated for the sizes smaller than $1 \times 10^{-9} \mathrm{~m}$. The diagram also shows a straight line (purple) corresponding to the elementary charge in $1 \mathrm{e}$.

Table 1 Dipole moments and energies available to molecules on the surface of the supercharged particles compared with the threshold of the thermal dissociation $E_{\text {act. }}$ for these molecules at $T=190 \mathrm{~K}$.

\begin{tabular}{lllllll}
\hline Characteristics of molecules & $\mathrm{ClOOCl}$ & $\mathrm{ClONO}_{2}$ & $\mathrm{O}_{3}$ & $\mathrm{HOONO}$ & $\mathrm{HO}_{2} \mathrm{NO}_{2}$ & $\mathrm{~N}_{2} \mathrm{O}_{5}$ \\
\hline $\mathrm{p}, \mathrm{D}$ & 0.72 & 0.77 & 0.53 & 1.07 & 1.99 & 1.39 \\
$\mathrm{~W}, \mathrm{eV}$ & 0.30 & 0.32 & 0.22 & 0.45 & 0.83 & 0.58 \\
$\mathrm{~W}+\mathrm{W}_{\mathrm{H} 2 \mathrm{O}}, \mathrm{eV}$ & 1.07 & 1.09 & 0.99 & 1.22 & 1.60 & 1.35 \\
$\mathrm{E}_{\mathrm{act}}, \mathrm{eV}$ & 0.69 & 0.90 & 1.00 & 0.87 & 0.94 & 0.95 \\
\hline
\end{tabular}

charge of 1e. The intersection of this straight line with the line corresponding to a solid particle determines the size of the maximal charged solid particle with a charge of 1e, which the size of is about $\sim 2.7 \AA$. Calculations show that such particle size is approximately of the same size as some ionic clusters.

For example, a positive core ion which frequently occurs in the atmosphere is $\mathrm{H}_{3} \mathrm{O}^{+}$[1]. $\mathrm{H}_{2} \mathrm{O}$ molecules and other molecules can attach to this core ion. The radius of $\mathrm{H}_{3} \mathrm{O}^{+}$is $\mathrm{r}_{1} \sim 1.0 \AA$ [28]. The calculated radius of water molecule is $\sim 1.7 \AA$ [29] and the experimental radius for this molecule is $\sim 1.4 \AA$ [29]. Thus, the distance between the centre of the ion and the water molecule is $\sim 2.4-2.7 \AA$, which is equal or even less than the radius of the supercharged particle with a charge of 1e. Other ions can also possess the properties of the supercharged particles in various degrees. Thus, the ions formed in the stratosphere naturally exhibit properties of the supercharged particles.

Table 1 lists the thresholds of the thermal dissociation for the series of compounds, as well as the energy available in the molecules of compounds in thermal dissociation reactions on the surface of a supercharged particle. $\mathrm{W}$ is the energy of a molecule of a compound on the surface of supercharged particles, $\mathrm{W}_{\mathrm{H} 2 \mathrm{O}}$ is the energy of a water molecule on the surface. $\mathrm{W}$ and $\mathrm{W}_{\mathrm{H} 2 \mathrm{O}}$ were calculated by Eq. (4), and the activation energy $E_{\text {act }}$ was determined on the 
basis of Refs. [30-32]. The temperature was assumed to be $\mathrm{T}=190 \mathrm{~K}$.

Table 1 shows that the energy available to molecules on the surface of supercharged particles is quite sufficient for their dissociation [21], taking into account the large effective concentration of water molecules and other species on the effective surface of the supercharged particle (see below).

Not only is the increase in energy important but also the increase of the concentration of molecules on the surface of the charged particle. Here, we come to the important part of our discussion, which permits a fresh look at the nature of the chemical reactions and the possibility of their occurrence on the surface of the charged particles.

According to the Boltzmann distribution, the concentration of molecules at a distance $r$ from a charged particle can be defined as:

$$
\mathrm{n}(\mathrm{r})=\mathrm{n}_{\infty} \exp \left(-\mathrm{W}_{0} / \mathrm{kT}\right),
$$

where

$$
\mathrm{W}_{0}=-|\mathrm{qp}| /\left(4 \pi \varepsilon_{0} \mathrm{r}^{2}\right)
$$

Considering Eqs. (3) and (4) and substituting $\mathrm{W}_{0}=$
$\mathrm{W}_{\max }$ into the Eq. (5), we can derive the concentration of the molecules at the surface of a supercharged particle with radius $r_{0}$ at $T=190 \mathrm{~K}$ :

$$
\mathrm{n}\left(\mathrm{r}_{0}\right)=\mathrm{n}_{\infty}(\exp (-0.415 / \mathrm{kT}))^{\mathrm{p}}=\mathrm{n}_{\infty}\left(1.04 \times 10^{11}\right)^{\mathrm{p}}
$$

Here, $\mathrm{kT}$ is expressed in $\mathrm{eV}$, and $\mathrm{p}$ is expressed in Debye units. According to Eq. (6), the concentration of compounds on a surface of the supercharged particle does not depend on its radius; therefore, calculations of concentrations in the vicinity of such particle can be carried out in terms of the size of the particle.

The results of the calculations of the concentrations of various substances on the surface of the supercharged particle according to Eq. (6) are given in Table 2. Similar calculations have been performed in our work [21] without the using of Eq. (6).

Fig. 3 shows the distribution of molecules of various compounds in the vicinity of the supercharged particles under conditions found in the lower stratosphere at the altitude of $20 \mathrm{~km}$. Concentrations of the specified compounds in ambient space are given in Table 2.

\begin{tabular}{|c|c|c|c|}
\hline Substance & $\begin{array}{l}\text { Concentration } \\
\text { in the volume, } \\
\text { molecules } / \mathrm{cm}^{3}\end{array}$ & $\begin{array}{l}\text { Dipole } \\
\text { moment, D }\end{array}$ & $\begin{array}{l}\text { Maximum concentration } \\
\text { on the supercharged particle } \\
\text { surface, molecules } / \mathrm{cm}^{3}\end{array}$ \\
\hline $\mathrm{H}_{2} \mathrm{O}$ & $5 \times 10^{12}$ & 1.85 & $1.2 \times 10^{33}\left(5 \times 10^{21}\right)$ \\
\hline $\mathrm{O}_{3}$ & $5 \times 10^{12}$ & 0.53 & $3.5 \times 10^{18}$ \\
\hline $\mathrm{HNO}_{3}$ & $2 \times 10^{10}$ & 2.17 & $1.6 \times 10^{34}\left(5 \times 10^{21}\right)$ \\
\hline $\mathrm{HCl}$ & $3 \times 10^{9}$ & 1.10 & $3.9 \times 10^{21}$ \\
\hline $\mathrm{ClONO}_{2}$ & $1 \times 10^{9}$ & 0.77 & $3.0 \times 10^{17}$ \\
\hline $\mathrm{ClO}$ & $1 \times 10^{9}$ & 1.30 & $2.1 \times 10^{23}\left(5 \times 10^{21}\right)$ \\
\hline $\mathrm{ClOOCl}$ & $1 \times 10^{9}$ & 0.72 & $8.6 \times 10^{16}$ \\
\hline $\mathrm{NO}_{2}$ & $1 \times 10^{9}$ & 0.32 & $3.4 \times 10^{12}$ \\
\hline NO & $1 \times 10^{9}$ & 0.16 & $5.8 \times 10^{10}$ \\
\hline $\mathrm{N}_{2} \mathrm{O}$ & $2 \times 10^{11}$ & 0.16 & $1.2 \times 10^{13}$ \\
\hline $\mathrm{N}_{2} \mathrm{O}_{5}$ (night) & $5 \times 10^{8}$ & 1.39 & $1.0 \times 10^{24}\left(5 \times 10^{21}\right)$ \\
\hline $\mathrm{HO}_{2} \mathrm{NO}_{2}$ & $8 \times 10^{7}$ & 1.99 & $6.7 \times 10^{29}\left(5 \times 10^{21}\right)$ \\
\hline HOONO & $8 \times 10^{7}$ & 1.07 & $4.9 \times 10^{19}$ \\
\hline $\mathrm{HOCl}$ & $3 \times 10^{7}$ & 1.30 & $6.3 \times 10^{21}\left(5 \times 10^{21}\right)$ \\
\hline $\mathrm{OH}$ & $5 \times 10^{5}$ & 1.65 & $7.5 \times 10^{23}\left(5 \times 10^{21}\right)$ \\
\hline $\mathrm{HO}_{2}$ & $2 \times 10^{6}$ & 2.09 & $2.1 \times 10^{29}\left(5 \times 10^{21}\right)$ \\
\hline
\end{tabular}

Table 2 The typical concentrations of various substances in the polar stratosphere at the altitude of $20 \mathrm{~km}$ and their concentrations on the supercharged particle surface for $T=190 \mathrm{~K}$.

Note: The estimate of the maximum permissible concentrations on the particle surface is given in the brackets, which correspond to the distance between the molecules of $\sim 6 \AA$. 


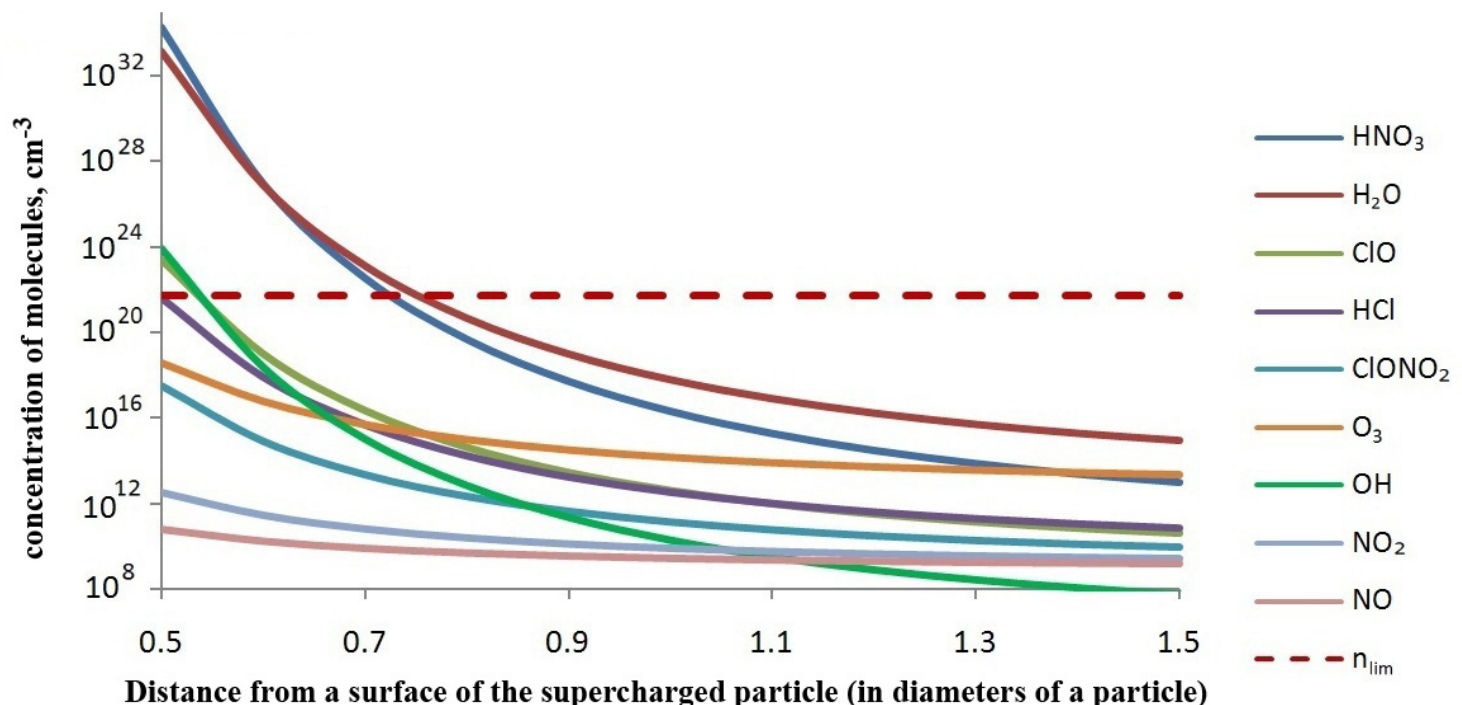

Fig. 3 Estimated concentrations of various compounds in the vicinity of the supercharged particles for lower stratospheric conditions at $T=190 \mathrm{~K}$. The dashed line shows the estimate of the maximum permissible concentration of molecules $\mathbf{n}_{\text {lim }} \sim 5 \times$ $10^{21}$ molecules $/ \mathrm{cm}^{3}$, corresponding to the minimum distance between molecules $\sim 6 \AA$. The concentrations of $\mathrm{H}_{2} \mathrm{O}, \mathrm{HNO}_{3}, \mathrm{OH}$ and CIO molecules which have large dipole moments exceed $\mathbf{n}_{\lim }$, while the increase in the concentrations of molecules with small dipole moments, such as $\mathrm{NO}_{2}$ or NO, is very small.

As seen from Eq. (6) and Fig. 3, the increased concentration of the molecules on the surface of the supercharged particles is strongly dependent on the dipole moment of the molecules. In this case, the calculated concentration of molecules with large dipole moments exceeds the maximum permissible concentration $\mathrm{n}_{\text {lim }}\left(\sim 5 \times 10^{21}\right.$ molecules $\left./ \mathrm{cm}^{3}\right)$, corresponding to the minimum possible distance between the molecules $\sim 6 \AA$. This applies to the molecules such as $\mathrm{H}_{2} \mathrm{O}$, with a dipole moment $\mathrm{p}$ of $\sim 1.85$ Debye (D), $\mathrm{HNO}_{3}(\mathrm{p} \sim 2.17 \mathrm{D}), \mathrm{OH}(\mathrm{p} \sim 1.65 \mathrm{D})$ and $\mathrm{ClO}(\mathrm{p} \sim 1.30 \mathrm{D})$. At the same time, the increase in the concentration of small molecules that have lower dipole moments such as $\mathrm{NO}_{2}(\mathrm{p} \sim 0.32 \mathrm{D})$ and NO $(p \sim 0.16 \mathrm{D})$ is very low. Note that the surface concentration of molecules with zero dipole moment does not increase according to the Boltzmann distribution. Therefore, the basic molecules that compose the atmosphere, such as $\mathrm{N}_{2}, \mathrm{O}_{2}$ and $\mathrm{CO}_{2}$, do not "adhere" to the surface of charged particles.

It should be pointed out that in some works [20, 33, 34] the adsorption of the non-polar molecules and atoms on the thin films charged by electrons was observed experimentally. Thus, in the work of Shamir et al. [33, 34] such effect was observed on the silicon surfaces for $\mathrm{He}, \mathrm{Ar}, \mathrm{N}_{2}, \mathrm{O}_{2}$, etc., and $\mathrm{Lu}$ [20] showed the similar effect on a thin ice surface for $\mathrm{CCl}_{4}$ molecules. Also, in the Lu's experiment [20] the simultaneous decomposition of a part of the $\mathrm{CCl}_{4}$ molecules was observed as a result of the electron capture by these molecules.

Indeed, the non-polar molecules and atoms can be polarized due to the electric field. However, the induced dipole moment of the molecules and atoms caused even by strong electric fields is rather small, and it is much smaller that the constant dipole moment of such molecules as $\mathrm{NO}$ and $\mathrm{NO}_{2}$, so we neglected it in our calculations. At the same time, as our calculations showed, under the stratospheric conditions not a single molecule of $\mathrm{NO}$, or $\mathrm{NO}_{2}$ can adhere to the surface of the extremely charged particle of the micrometer size range. But still we do not exclude the possibility of the non-polar molecules adsorption in the stratosphere through the process of the capturing of the electron by the molecule, i.e., at first the molecule captures the electron on the charged 
surface, and then the ion is adsorbed with the possibility of its subsequent decomposition. Yet it is a different process, which has no relation to the dipole interaction of the neutral molecules with the charged particles.

It is worth mentioning also that for the positively charged surfaces such "sticking" effect of non-polar molecules is not observed.

Despite a significant concentration of molecules on the surface of the supercharged particles, especially molecules with the large dipole moments, the total number of adherent molecules (within a "sticking" radius) is not very large. According to Ref. [21], the number of water molecules on the surface of the maximal charged irregularity of the micron size can reach $\sim 7 \times 10^{9}$, the number of molecules of $\mathrm{ClO} \sim 7 \times$ $10^{8}$, and the number of molecules of $\mathrm{NO}_{2}$ and $\mathrm{NO}$ are 0.5 and 0.03 , respectively. As the size of the charged particle decreases, the number of "sticky" molecules decreases, and at the same size as the size of the ion, a number of molecules on the order of tens of molecules with a high dipole moment-primarily water molecules - might remain on the effective surface.

Now imagine that complicated molecules impact the surface of a supercharged particle, so they can decay on the surface into simpler molecules. The products of this decay may behave in different ways. For example, the dissociation of $\mathrm{ClONO}_{2}$ results in $\mathrm{ClO}$ and $\mathrm{NO}_{2}$ on the surface and these two molecules have the significantly different from each other dipole moments.

According to our calculations, no $\mathrm{NO}_{2}$ molecules should "stick" and remain on the surface, i.e., $\mathrm{NO}_{2}$ molecules are ejected into ambient space while the $\mathrm{ClO}$ molecules "stick" and remain on the surface. Subsequently, the $\mathrm{ClO}$ molecule can participate in other reactions on the surface. The analysis of the possible chemical reactions with the participation of the charged particles will be considered below.

Now, we proceed to the analysis of experiments in the Polar Regions to find an evidence for the role of charged particles in the polar stratosphere.

\section{The Role of Ions in Chemical and Electrical Processes in the Polar Stratosphere}

By the end of the 1980s, Hoffmann and colleagues [35], using balloon measurements, discovered a decrease of the ozone concentration that formed over the aerosol layer in the cold polar stratosphere. Moreover, the increased content of condensation nuclei was observed in the ozone depleted area. The upper part of Fig. 4 shows the altitude profiles of the aerosol concentrations over Kiruna $\left(68^{\circ} \mathrm{N}, 20^{\circ} \mathrm{E}\right)$ on 23 January and 30 January 1989, the ozone mixing ratio on 23 January 1989 [35], as well as the altitude profiles of the condensation nuclei in the Polar Regions in winter and spring, including the measurement over Kiruna on 30 January 1989 [36].

Why is there an increase in the concentration of condensation nuclei on the upper boundary of the aerosol layer, which according to Ref. [37] is the part of PSCs?

Hofmann and colleagues suggested that it was the increasing mono-nuclearization and the progression of specific reactions involving sulphates occur $[36,38$, 39]. However, we assumed that the increase in the number of condensation nuclei on the cloud top was associated with the work of the global electric circuit and the accumulation of charged particles at the upper boundary of the PSCs. Perhaps, those particles also caused a depression of the ozone. The question of precisely how the ozone was depleted and the role of chlorine compounds in this destruction will be addressed later.

Renard et al. [40] were the first who performed the direct measurements of charged aerosols in the polar stratosphere. Figs. $4 \mathrm{~d}$ and $4 \mathrm{e}$ show the altitude profiles of aerosol concentrations, including charged aerosols, as well as the proportion of the charged aerosols at different altitudes [40]. These figures show that the largest number of charged particles is observed on the upper and lower aerosol boundaries. 

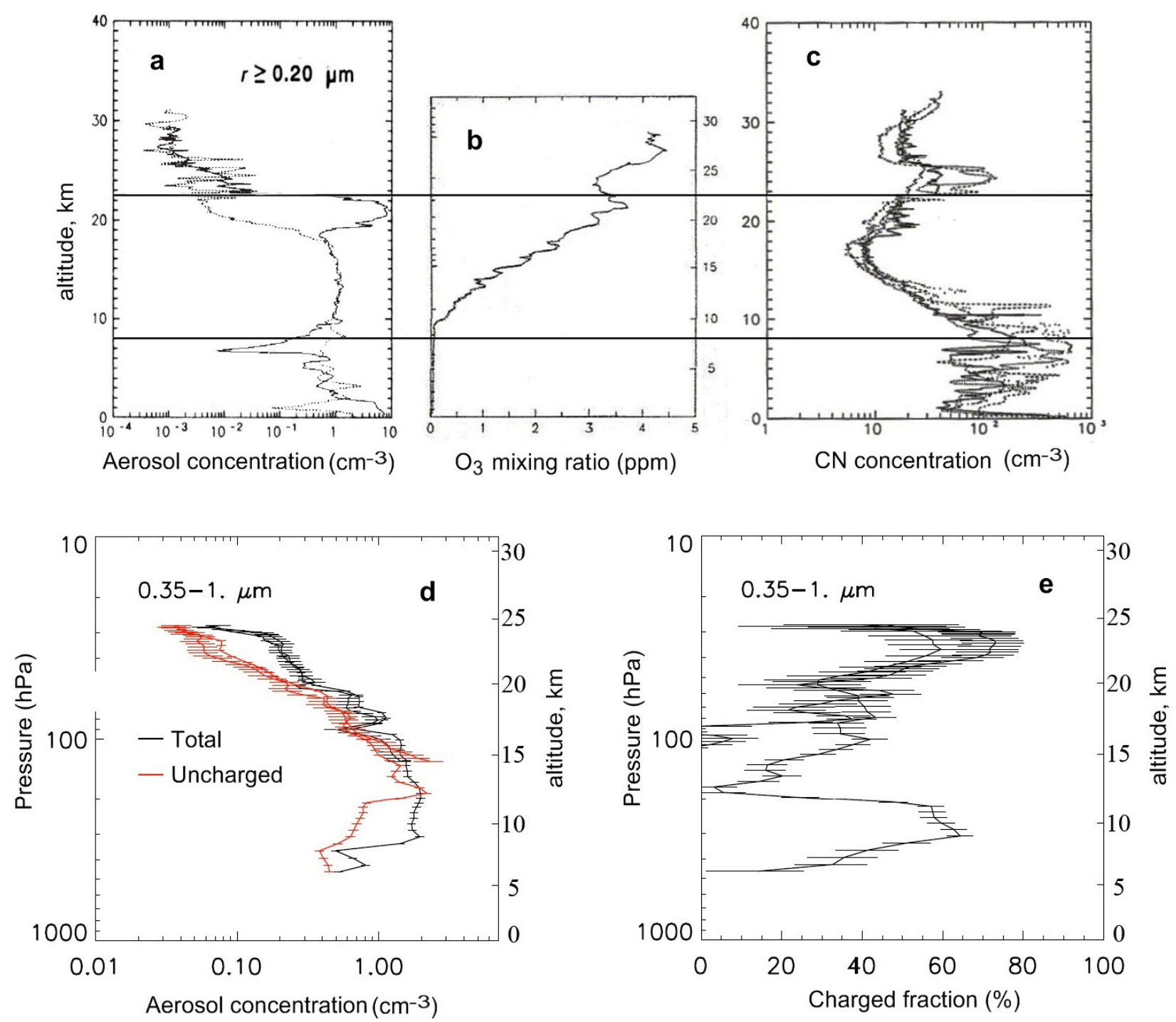

Fig. 4 Balloon measurements in the polar stratosphere (from our work [22]). Vertical altitude profiles of the aerosol concentrations over Kiruna on 23 January (full line) and 30 January (dashed lines) 1989 [35] (a) and ozone mixing ratio over Kiruna on 23 January 1989 [35] (b). The concentration of the condensation nuclei over Kiruna on 30 January 1989 (solid line, c) and the concentration of condensation nuclei in the Antarctic at $7^{\circ}$ S. Lat..: .... -August 27, 1988; ...........-September 6, 1988; ........-. September 14, 1988 (c) [36]; altitude profiles of the total concentration of aerosols and the uncharged fraction of aerosols in the size range 0.35-1 $\mu \mathrm{m}$ (d), as well as the proportion of the charged aerosol particles during the ascent and descent of the balloon over Kiruna on 12 March 2011 (e) [40].

Measurements in that balloon experiment were carried out by a special aerosol counter. At the entrance of this counter, an electric trap was installed. This electric trap does not let charged particles pass inside the counter. Unfortunately the charge sign of the aerosols was not distinguished in this experiment.

The significant decrease in the distribution of the condensation nuclei concentration may be linked to their transformation into PSC drops and also to the adhesion of the condensation nuclei to water drops and aerosol particles. As this significant decrease is associated with PSCs, we are convinced that it disappears in the summer, when no PSCs exist [41].

As shown previously, for the charged particles, the ions that are the most apt to destroy the ozone are those that are the most similar in properties to the 
supercharged particles (see Fig. 2, Table 1). Therefore, we hypothesized that ozone depletion on the upper PSC boundary, as well as the increase in the number of condensation nuclei, is mainly due to the increase of ions in this area. At the same time, the nature of the altitude distribution of the charged aerosols is associated with the peculiar high-altitude distribution of the ions resulting from the global electric circuit work in the presence of PSCs and aerosol particles.

The global electric circuit is described in detail in the literature [42-44]. We shall mention only the specific aspects of its functioning. In the atmosphere, the presence of ions and the electric field in the stratosphere cause the streaming of small currents on the order of several pA per square metre $[45,46]$.

The balanced average concentration of ions in the absence of clouds is shown in Fig. 5 [2]. As a rule, we see a rise in the ion concentration with a decrease of altitude in the range $\sim 10$ to $50 \mathrm{~km}$, while the calculations of the ion concentration itself are weakly dependent on the presence of background aerosols to which the ions "stick" (Fig. 5). However, this "sticking" becomes significant in the presence of a cloud (Fig. 6).

As the conductivity inside the cloud decreases, the charged particles accumulate on the cloud boundaries: the positive ones on the top, and the negative ones on the bottom (Fig. 6) [45]. These charged particles lead to the generation of an additional electrical field, and the ions inside the cloud move more quickly. Currents inside and outside of the clouds are aligned in such way that the further accumulation of the charge at the cloud boundaries is prohibited (see Fig. 8 below). Those effects have been reproduced by the contemporary models and observations in the troposphere [45, 47, 48].

Note that inside the cloud, the concentration of the positive and negative ions are practically the same, which is the reason why the charged aerosols that have formed are quasi-neutral and their charges are neutralised. At the same time, the positively charged aerosols are produced on the cloud top while negatively charged aerosols are present on the lower boundary of the cloud because of the presence of excessive amounts

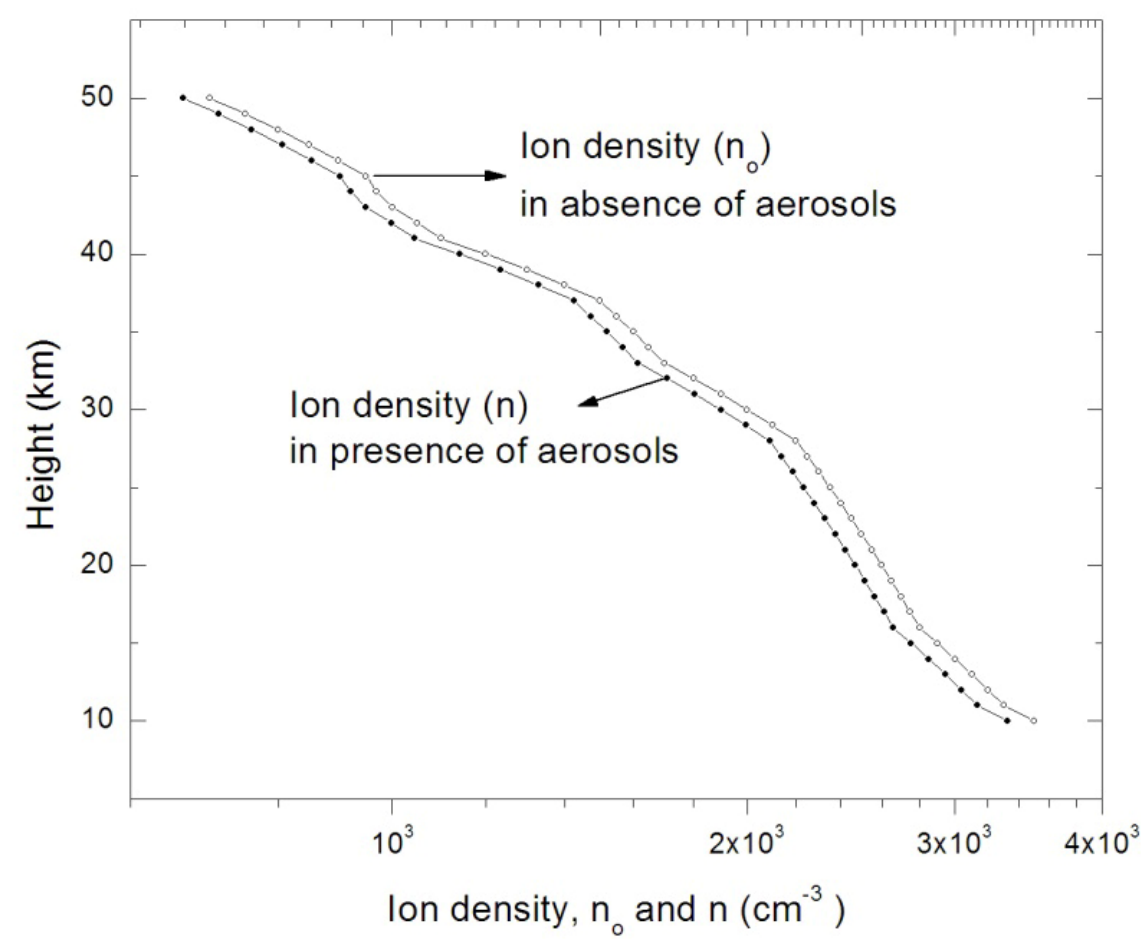

Fig. 5 The average altitude profile of the ion distribution in the atmosphere, with and without the consideration of background aerosols [2]. 


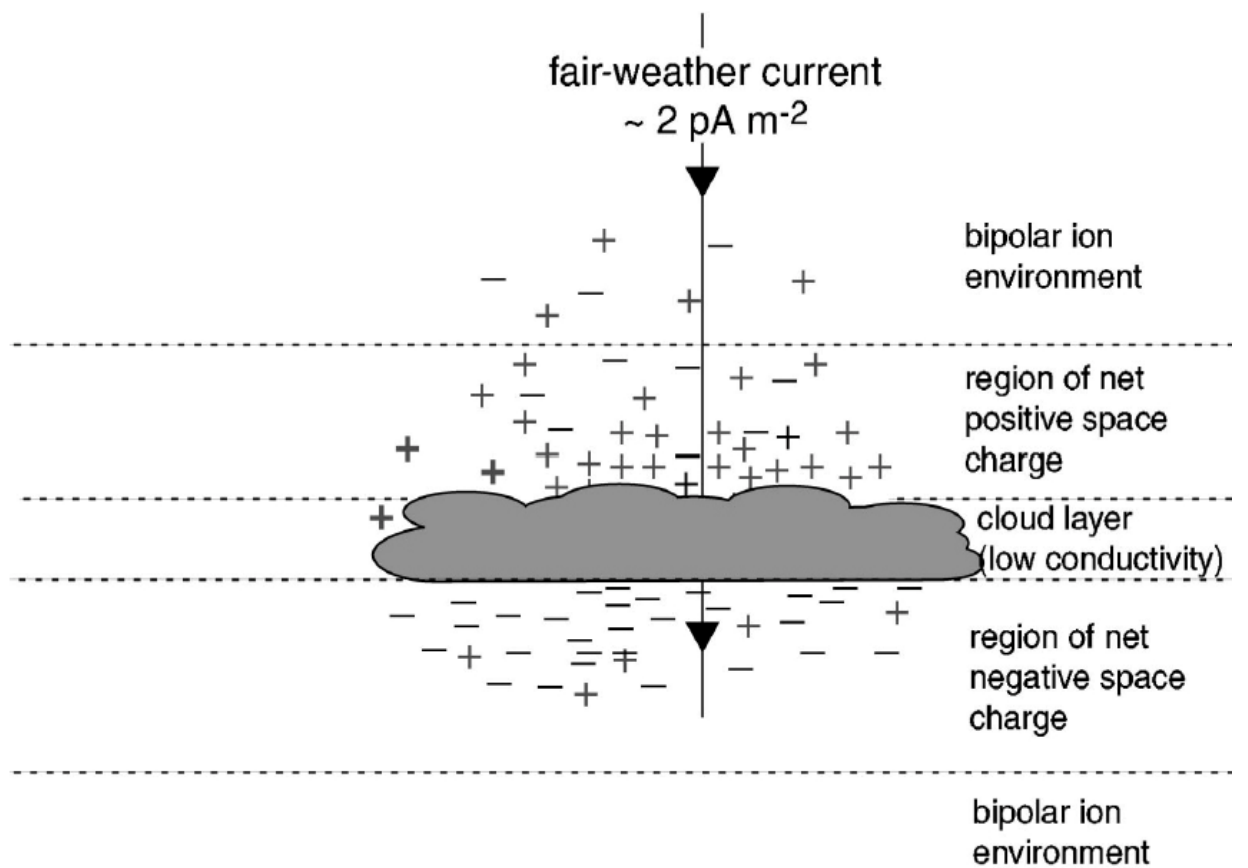

Fig. 6 The distribution of ions and charged particles in the presence of the cloud in the troposphere as a result of the global electric circuit work [45]. The increased concentrations of positive and negative ions are observed above the upper and below the lower cloud boundaries, respectively. In addition, in the real cloud layer, a low concentration of ions can be observed because of their adhesion to water drops.

of ions of the corresponding sign. This explains the results of the measurements of charged aerosols, as shown in Figs. 4d and 4e.

Estimates of the discussed effects have been made earlier for tropospheric clouds [45, 47, 48]. Do those effects vary significantly under stratospheric conditions?

For answering this question, we performed a series of calculations. A change in the concentration of the positive and negative ions in the atmosphere is determined by the following equation:

$$
\frac{d n}{d t}=q-\alpha n^{2}-k n,
$$

where $\mathrm{n}$ is the ion concentration of a particular sign, $\mathrm{q}$ is the rate of formation of ions, $\alpha$ is the coefficient of the recombination of the positive and negative ions, and $\mathrm{k}$ is the coefficient for the sink of ions determined by various factors, which we shall subsequently consider.

Under equilibrium conditions, $\mathrm{dn} / \mathrm{dt}=0$, and then:

$$
n=\frac{\sqrt{k^{2}+4 \alpha q}-k}{2 \alpha}
$$

If $\mathrm{k}=0$, i.e., if there is no ion sink except for recombination, then,

$$
n=\sqrt{\frac{q}{\alpha}}
$$

Now we consider possible additional ion sinks. The adherence of ions to background aerosols was the focus in a number of studies [2, 45, 49], and it was not found to provide a significant change in the altitude distribution of ions (Fig. 5).

At the same time, the sticking coefficient of the ions to water drops is much higher than that one to the background aerosols. Under the tropospheric conditions, this coefficient is calculated via the ion diffusion coefficient [50, 51]. Under stratospheric conditions, we can estimate the sticking coefficient of the ions to the drops using an approximation of the theory of "collisions" [52]:

$$
k=P S_{0} v n / 4,
$$

where $\mathrm{P}$ is the probability of the ion sticking to the drop, $S_{0}$ is the surface area of the droplet, $n$ is the concentration of drops, and $\mathrm{v}$ is the average velocity of the ions. 
Eq. (10) assumes the existence of a monodispersed size particles distribution. There, we can determine the area of drop per unit volume as $\mathrm{S}=\mathrm{S}_{0} \mathrm{n}$. If the distribution in Eq. (10) is not the monodispersed one, $\mathrm{S}$ can be defined as the sum of squares of all particles per unit of volume.

Fig. 7 shows the results of calculations of the equilibrium concentration of ions according to Eqs. (8-10) when a homogeneous cloud is present in the stratosphere in the altitude range of $18-22 \mathrm{~km}$. In this altitude range of $18-22 \mathrm{~km}$ the ion concentration was calculated by Eq. (7), taking into account the sticking coefficient $\mathrm{k}$ as in Eq. (10). Outside this altitude range where $\mathrm{k}=0$, Eq. (9) was used for calculations.
The calculations were carried out for various values of $\mathrm{S}$. The effective radii of the particles corresponding to a particular $\mathrm{S}$ value are also indicated. The coefficient $\mathrm{k}$ was calculated by Eq. (10), where $\mathrm{P}=1$ and $\mathrm{v}=3 \times 10^{4} \mathrm{~cm} / \mathrm{s}$. In the calculations, we assumed that $\mathrm{q}=5 \mathrm{~cm}^{-3} \mathrm{~s}^{-1}$ and $\alpha=1.6 \times 10^{-6} \mathrm{~cm}^{3} \mathrm{~s}^{-1}$, the values for $20 \mathrm{~km}$ are applicable to all altitudes. Nevertheless, such an approach allows to make a correct estimation of the decrease in the altitudinal distribution of ions at the altitude of $\sim 20 \mathrm{~km}$ in the case of a small geometrical depth of the aerosol layer.

Note that the probability $\mathrm{P}=1$ corresponds to the maximum possible absorption coefficient under those conditions and the accepted average velocity of the ions which is typical for the light ions, such as $\mathrm{HO}_{3}{ }^{+}$.

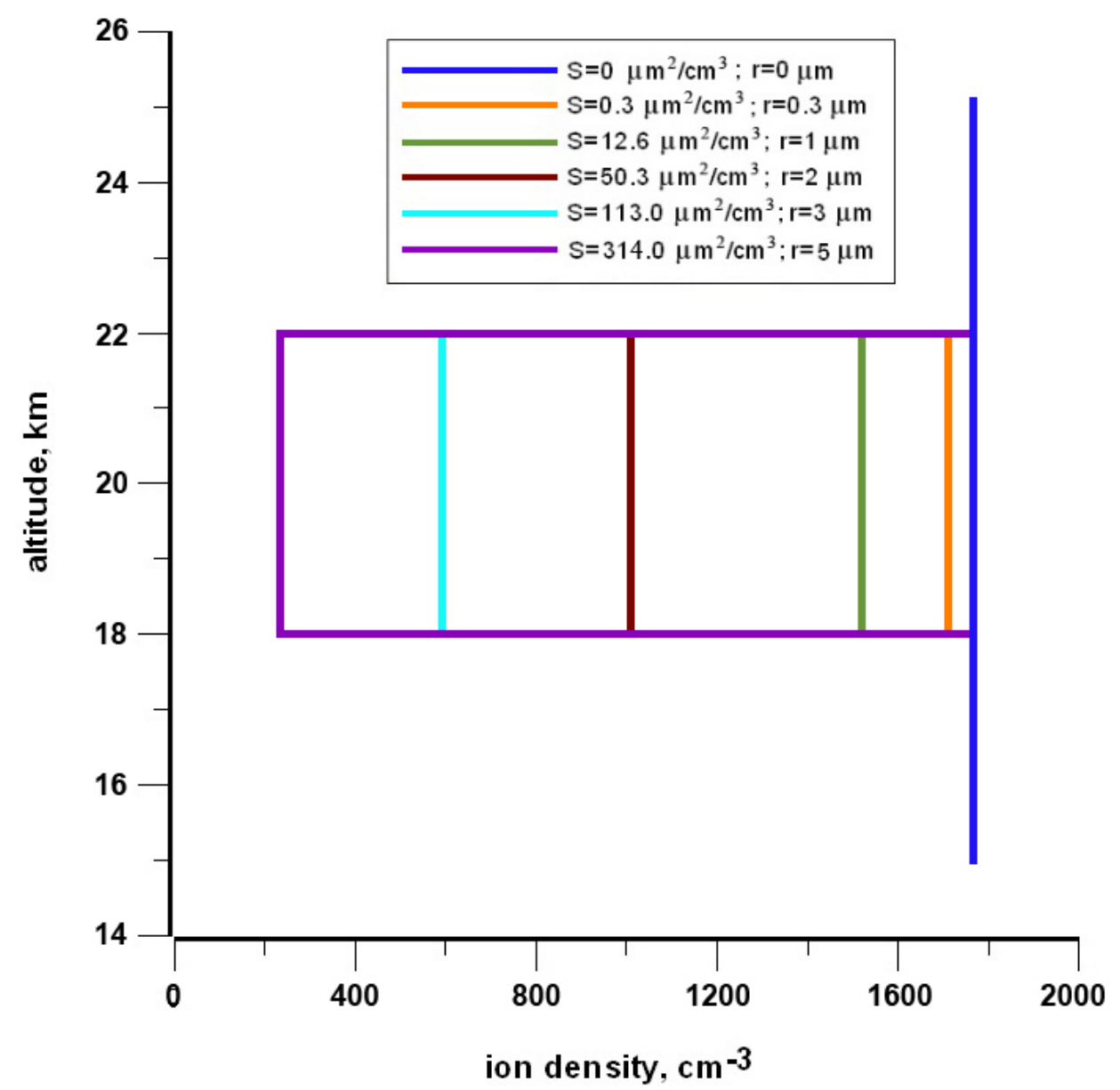

Fig. 7 The results of the calculation of the ion concentration in the presence of a cloud in the stratosphere with various areas of drops (S) per unit volume. The effective drop size ( $r$ ), with an equivalent area $S$ is also shown. The cloud, composed of liquid drops, is located at the altitudes of $18-22 \mathrm{~km}$, and the concentration of drops inside the cloud is assumed to be constant. 


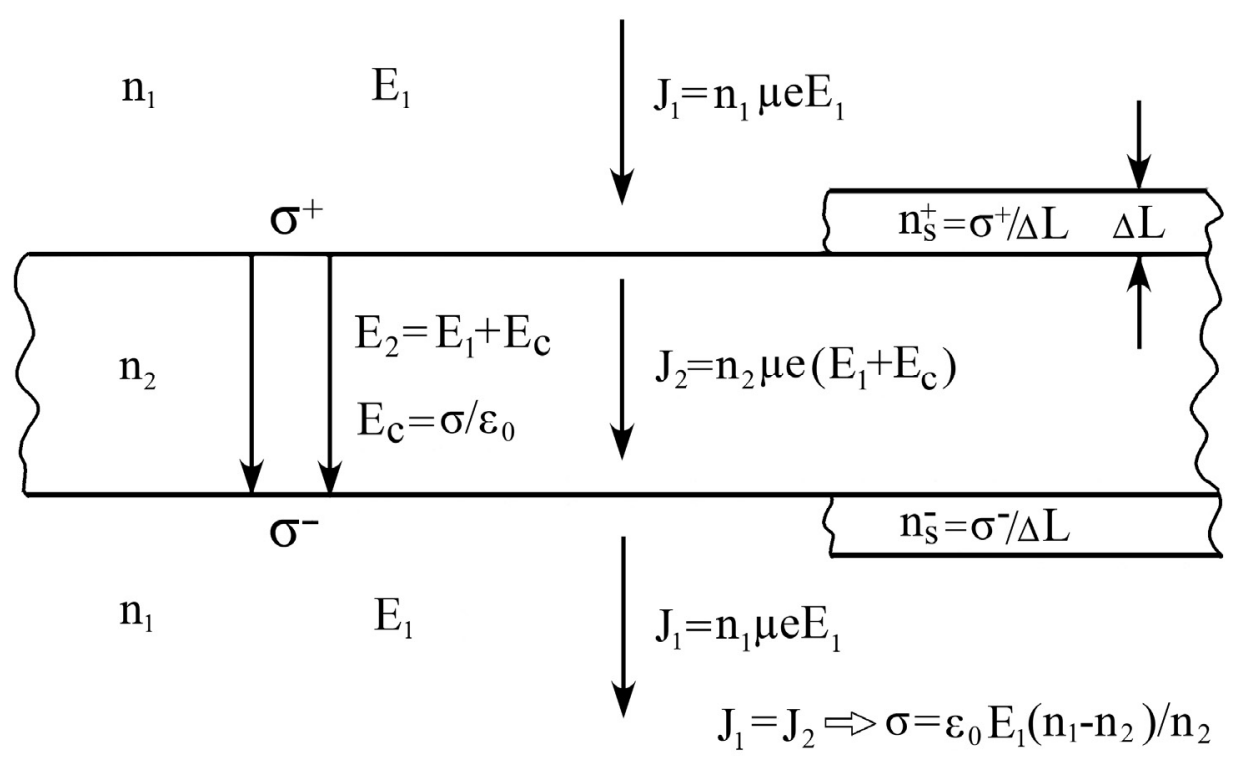

Fig. 8 The unipolar charge accumulation on the cloud boundaries is shown as a part of the global electric circuit. The stationary work of this circuit is ensured if the current densities outside the cloud $J_{1}$ and inside the cloud $J_{2}$ are equal. A reduced concentration of ions inside the cloud $n_{2}$ compared to the concentration of ions outside the cloud $n_{1}\left(n_{2}<n_{1}\right)$ is compensated by their more rapid movement. This movement allows the accumulation of a surface charge ( $\sigma)$ on the boundaries of the cloud. As a result of those processes, the electric currents inside and outside of the clouds are balanced.

As observed from Fig. 7, a significant decrease in the altitudinal distribution of ions can be as high as an order of magnitude with particle sizes of a few microns. At the same time, the estimates show that even for a large area of drops, the amount of the accumulated unipolar space charge at the boundaries of the cloud is extremely small. We shall consider this issue in more detail.

Fig. 8 shows the scheme of the work of the global electric circuit when there is a homogeneous cloud with a constant concentration of drops. This scheme permits to estimate the value of a unipolar charge on the boundaries of the cloud.

Fig. 8 shows the equilibrium ion concentrations $\left(\mathrm{n}_{1}\right.$ and $\mathrm{n}_{2}$, respectively) outside and inside the cloud. Here, $n_{1}$ corresponds to the solution (9) of the Eq. (7) and $n_{2}$ to the solution (8) of the equation (7) for the given coefficient $\mathrm{k}$, corresponding to the particular drop size. If the electric field $E_{1}$ exists in the stratosphere, then the electric current will be result of it. The initial density of this current is different inside and outside the cloud because of the different ion concentrations in those areas. Outside the cloud, the current density is $\mathrm{J}_{1}=\mathrm{n}_{1} \mu_{1} \mathrm{eE}_{1}$, while inside the cloud, it is $J_{2}=\mathrm{n}_{2} \mu_{2} \mathrm{eE}_{1}$, where $\mu_{1}$ and $\mu_{2}$ are the ion mobilities inside and outside the cloud, respectively.

We assumed in the calculations that the mobility of the ions did not change with the altitude and was the same both inside and outside the cloud, wherein $\mu_{1}=$ $\mu_{2}=\mu$. Because $J_{1}>J_{2}$, then this leads to an influx of positive ions (outflow of negative ions) on the upper boundary and a change in the magnitude of the electric field inside the cloud. Similarly, a negative charge accumulates at the lower boundary of the cloud.

Obviously, the accumulation of a unipolar charge on the boundaries of the cloud cover is accompanied by the emergence and increase in field $\mathrm{E}_{\mathrm{C}}$ within the cloud, which is close to the condenser field $\mathrm{E}_{\mathrm{C}}=\sigma / \varepsilon_{0}$, where $\sigma=\sigma^{+}=\sigma^{-}$(the surface charge density), and $\varepsilon_{0}$ is the electric constant. The resultant field inside the cloud $\left(E+E_{C}\right)$ increases the speed of the ions and the current $\left(\mathrm{J}_{2}\right)$ inside the cloud. The accumulation of charge on the boundaries of the cloud will continue 
until the currents outside and inside the cloud are balanced. Based on the condition $\mathrm{J}_{1}=\mathrm{J}_{2}$ or $\mathrm{n}_{1} \mu_{1} \mathrm{eE}_{1}=$ $\mathrm{n}_{2} \mu_{2} \mathrm{e}\left(\mathrm{E}_{1}+\sigma / \varepsilon_{0}\right)$, we can determine the surface density of the unipolar electric charge that has accumulated at the boundaries of the cloud:

$$
\sigma=\varepsilon_{0} \mathrm{E}_{1}\left(\mathrm{n}_{1}-\mathrm{n}_{2}\right) / \mathrm{n}_{2}
$$

The electric field of the surface charge can be represented as a thin layer field of the space charge with thickness $\Delta \mathrm{L}$, and the volume of the unipolar space charge concentration $n_{s}$ can be defined as:

$$
\mathrm{n}_{\mathrm{s}}=(1 / \Delta \mathrm{L}) * \varepsilon_{0} \mathrm{E}_{1}\left(\mathrm{n}_{1}-\mathrm{n}_{2}\right) / \mathrm{n}_{2}
$$

Table 3 shows the results of calculations of $\sigma$ and $n_{s}$ according to Eqs. (11) and (12) for a variety of effective drop sizes. The equilibrium ion concentrations $n_{1}$ and $n_{2}$ were calculated according to Eqs. (8-10). In this case, the charge is defined in elementary charges (e). In Eqs. (11) and (12), the value of the electric field $\mathrm{E}_{1}$ was assumed to be 0.5 $\mathrm{V} / \mathrm{m}$ [53]. Table 3 shows that the space density of the accumulated charge is extremely low even for the large particle sizes. At this concentration of space charge, it is unlikely to see a substantial increase in the condensation nuclei for the cloud (if it is associated with ions), or an increase in the charge of aerosols at the cloud boundaries (Fig. 4). Therefore, we assume the existence of an additional mechanism, which results in a substantial increase in the unipolar space charge at the cloud boundaries.

According to Eq. (8), with an infinite increase in coefficient $\mathrm{k}$, the concentration $\mathrm{n}_{2}$ tends to zero, and the volume concentration $n_{s}$ on the cloud boundaries will tend to infinity. According to Eq. (10), an increase in $\mathrm{k}$ can indicate an increase in the specific area of particles, but the area of PSC particles is usually not more than $\sim 100 \mu \mathrm{m}^{2} / \mathrm{cm}^{3}$ [54]. As observed from Table 3, even for such a particle area, the volume concentration of the charge is small (explanations for the last line in this table will follow). At the same time, the ion sink can be triggered not only by the process of the attachment of ions to the drops. The process of the direct formation of drops on ions is also possible.

However, what is the probability for the condensation of liquid on the ions? We shall consider this issue to some detail.

\section{The Condensation of Liquids on Ions}

The condensation of water vapour on ions is observed in a Wilson chamber [55]. However, to enact this process using calculations made on the basis of the thermodynamic theory of J. W. Gibbs [26], a high degree of vapour supersaturation $(\sim 4)$ is required (Fig. 9). At the same time some results $[4,56]$ and our calculations indicate that the condensation of water vapour and other liquids does not require such a strong supersaturation. We explain this important result according to our interpretation. As it is well-known, an equilibrium vapour pressure increases over the convex surface of a phase boundary. According to the Kelvin equation, the degree of saturation of liquid vapours (S) at a temperature (T) required for the condensation on a drop of radius $r$ is determined as follows [26]:

$$
\mathrm{S}=\mathrm{P} / \mathrm{P}_{\infty}=\mathrm{n} / \mathrm{n}_{\infty}=\exp (2 \gamma \mathrm{M} / \rho \mathrm{RTr})
$$

\begin{tabular}{|c|c|c|c|c|}
\hline $\begin{array}{l}\mathrm{S}, \mu \mathrm{m}^{2} / \mathrm{cm}^{3} \\
(\mathrm{r} \mu \mathrm{m})\end{array}$ & $\sigma$, quantity e $/ \mathrm{cm}^{2}$ & $\begin{array}{l}\mathrm{n}_{\mathrm{s}}, \text { quantity e/ } \mathrm{cm}^{3} \\
\Delta \mathrm{L}=100 \mathrm{~m}\end{array}$ & $\begin{array}{l}\mathrm{n}_{\mathrm{s}}, \text { quantity e/cm } \\
\Delta \mathrm{L}=1,000 \mathrm{~m}\end{array}$ & $\begin{array}{l}\mathrm{n}_{\mathrm{s}}, \text { quantity } \mathrm{e} / \mathrm{cm}^{3} \\
\Delta \mathrm{L}=3,000 \mathrm{~m}\end{array}$ \\
\hline $0.3(0.05)$ & $1.99 \times 10^{2}$ & $1.99 \times 10^{-2}$ & $1.99 \times 10^{-3}$ & $6.63 \times 10^{-4}$ \\
\hline $12.6(1.0)$ & $8.82 \times 10^{2}$ & $8.82 \times 10^{-2}$ & $8.82 \times 10^{-3}$ & $2.94 \times 10^{-3}$ \\
\hline $50.3(2.0)$ & $4.18 \times 10^{3}$ & $4.18 \times 10^{-1}$ & $4.18 \times 10^{-2}$ & $1.39 \times 10^{-2}$ \\
\hline $113(3.0)$ & $1.10 \times 10^{4}$ & 1.10 & $1.10 \times 10^{-1}$ & $3.68 \times 10^{-2}$ \\
\hline $314(5.0)$ & $3.62 \times 10^{4}$ & 3.62 & $3.62 \times 10^{-1}$ & $1.21 \times 10^{-1}$ \\
\hline $\begin{array}{l}\mathrm{t}=\left(1 \times 10^{-3}-1 \times 10^{-2}\right) \mathrm{s} \\
(\text { see text })\end{array}$ & $2 \times 10^{8}-2 \times 10^{9}$ & $2 \times 10^{4}-2 \times 10^{5}$ & $2 \times 10^{3}-2 \times 10^{4}$ & $2 \times 10^{2}-2 \times 10^{3}$ \\
\hline
\end{tabular}

Table 3 The unipolar surface charge density and the equivalent density of the unipolar space charge accumulated at the cloud boundaries in the stratosphere for different surface sizes of drops per unit volume inside the cloud. 
Here, $\mathrm{P}$ and $\mathrm{P}_{\infty}$ are the pressure of the vapour over the drop and over the planar surface, $n$ and $n_{\infty}$ correspond to the pressures of the concentration of the liquid molecules, $\gamma$ is the coefficient of the surface tension of the liquid, $\mathrm{M}$ is the molecular weight of the liquid, $\rho$ is the density of the liquid, $R$ is the universal gas constant, and $\mathrm{T}$ is the ambient temperature in Kelvin degrees.

At the same time, if the concentration of liquid vapour molecules in the space is $\mathrm{n}_{\infty}$, then according to Eq. (5), the relative number of molecules in the ion environment is determined by the distribution:

$$
\mathrm{n}_{\mathrm{ion}} / \mathrm{n}_{\infty}=\exp (-\mathrm{W} / \mathrm{kT})
$$

where

$$
\mathrm{W}=-|\mathrm{qp}| \cos \theta /\left(4 \pi \varepsilon_{0} \mathrm{r}^{2}\right)
$$

Eq. (14) is a generalized version of Eq. (5) for the case when the dipole orientates an angle $\theta$ with respect to the electric field vector. Statistically, the average value of $\langle\cos \theta>$ can be calculated with the help of the Langevin function $<\cos \theta>=\mathrm{L}(\mathrm{x})=$ cth $\mathrm{x}-$ $1 / x$, where $x=-W_{0} / k T=|q p| /\left(4 \pi \varepsilon_{0} r^{2} k T\right)$.

This means that if we build an imaginary spherical surface of radius $r$ around the ion, the degree of saturation $\left(\mathrm{S}_{\text {ion }}\right)$ required for vapour condensation on this surface will be determined by the ratio of Eqs. (13) and (14):

$$
\mathrm{S}_{\text {ion }}=\exp (2 \gamma \mathrm{M} / \rho \mathrm{RTr}) / \exp \left(|\mathrm{qp}| \cos \theta /\left(4 \pi \mathrm{kT} \varepsilon_{0} \mathrm{r}^{2}\right)\right)
$$

In reality, such a surface does not exist, but there could be a spherical drop of radius $r$, the surface of which is a liquid-gas interface. It is important that the surface of such an interface in the gas concentration is also given by Eq. (14) and the degree of saturation necessary for the condensation process is determined by Eq. (15). This conclusion follows because the field of the dipole molecules of a liquid disposed symmetrically around the ion is practically zero in a gaseous medium, including that one at the surface of the drop. Thus, the ion field is not shielded by the liquid environment in the gaseous medium. It is correct at least for the droplet size at which the
Langevin function is close to 1 for all molecules inside a droplet. According to our estimates, $\mathrm{L}(\mathrm{x})=$ 0.9 for molecules on the droplet surface of approximately $8 \AA$ in size (radius). This value may be considered as a conditional borderline below which the symmetry of the molecules should be exposed to their full extent.

Fig. 9 shows similar calculations for mixtures of water and surface-active agents (SAS, surfactants) [57]. Small amounts of added surfactants (i.e., $\sim 0.01-1 \%$ by mass) can reduce the surface tension of water from $\sim 73 \times 10^{-3}$ to $\sim 25 \times 10^{-3} \mathrm{~J} / \mathrm{M}^{2}$. In our opinion, $\mathrm{HNO}_{3}$ can serve as such a surfactant because its interaction with hydroxyl radical (see below) forms $\mathrm{NO}_{3}$ molecules, which have a zero dipole moment. These molecules are concentrated at the drop's surface, weakening the surface tension of the liquid. Calculations show that the supersaturation necessary for the formation of mixtures of surfactants is only $\sim 107 \%$.

Drops and ice particles can form in the following way. As Fig. 3 shows, a quasi-solid or quasi-liquid nucleus from water molecules and other molecules exists on the effective surface of an ion (perhaps this is the first layer of the molecules). It's estimated that concentration is $\sim 5 \times 10^{21}$ molecules $/ \mathrm{cm}^{3}$, which is characteristic of a liquid body or a solid material. The growth of a droplet, if conditions allow, begins with the attachment of molecules to this nucleus, and its size increases as the temperature decreases.

According to Refs. [58, 59], with a temperature decrease in the stratosphere and mesosphere, the number of water molecules $\mathrm{n}$ composing the $\left(\mathrm{H}_{3} \mathrm{O}^{+}\right)\left(\mathrm{H}_{2} \mathrm{O}\right)_{\mathrm{n}}$ cluster rises from 3-6 to 10 or higher. It is the authors' opinion $[58,59]$ that ion clusters with a large number of ligands of $\mathrm{H}_{2} \mathrm{O}$ molecules (presumably $\mathrm{n}>10$ ) provide the basis for cloud particles.

If the saturated vapour pressure of liquid does not exceed the threshold shown in Fig. 9 (for example, the growth of droplets starts from point A), small droplets 


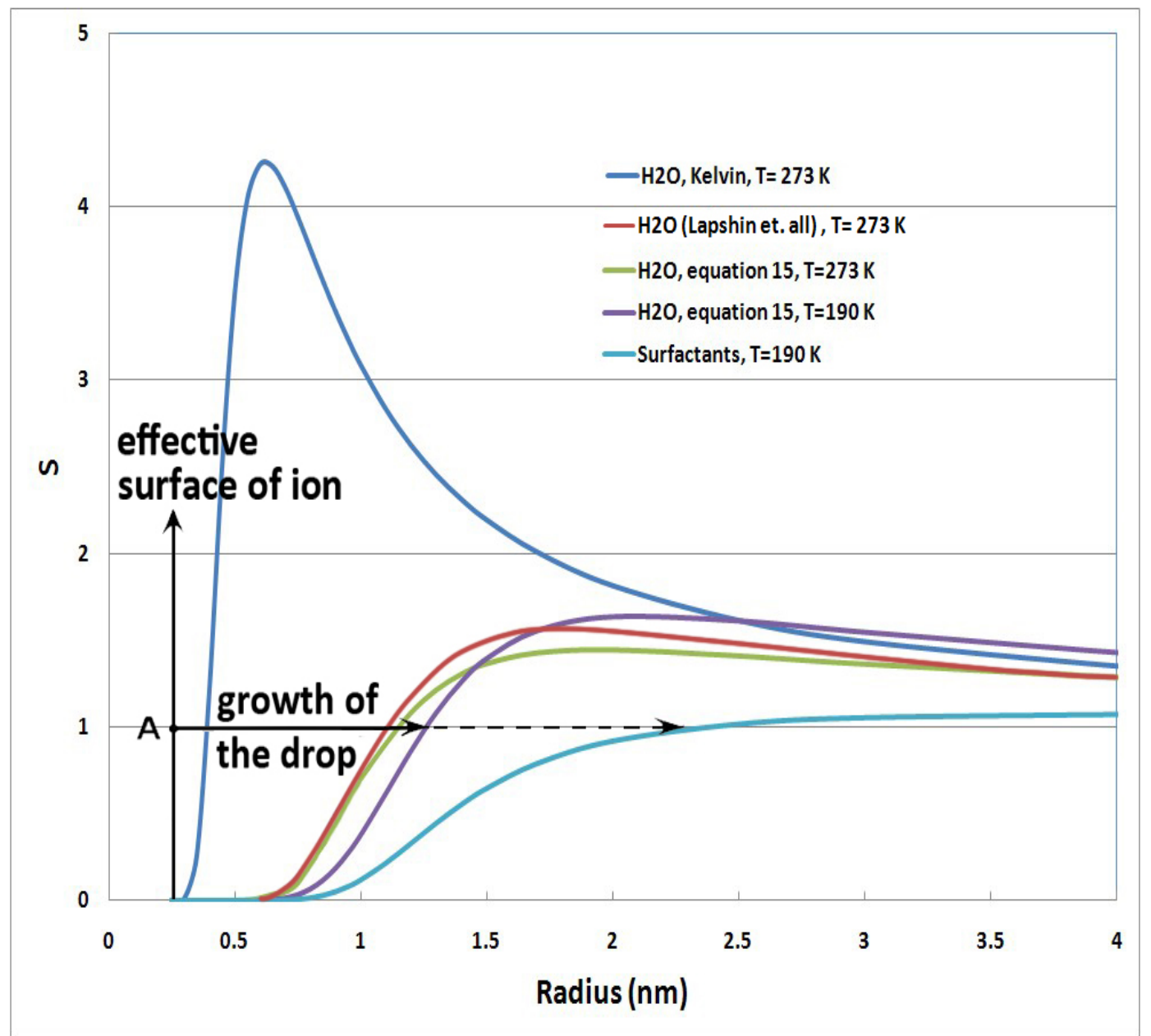

Fig. 9 The saturation of liquids $\mathrm{S}$, required for vapour condensation on a drop with a central monovalent ion. The estimates of the degree of saturation of water vapour are made according to Kelvin for $273 \mathrm{~K}$ [26], according to Ref. [4] and according to Eq. (15) for $273 \mathrm{~K}$ and $190 \mathrm{~K}$. The latter calculations apply to water vapour with added surfactants. The growth of drops is shown at liquid saturations of $100 \%$ (see text).

of nanometre size appear, some of which may turn into ice particles. If saturation exceeds the maximum for the corresponding liquid, then the unlimited growth of the drop will begin, which in actuality stops quickly because large drops of liquids can change shape under the influence of external forces, resulting in a loss of symmetry that reduces the electric field of the ion on the surface of the liquid-gas interface and halts the growth of the drop. For this reason, experimental observations of the condensation on ions can be very different from calculations because of the breakdown in symmetry of the drop due to external forces such as the inhibitory action of the ion, the influence of strong magnetic fields, etc.
Similarly, when a drop freezes, its spherical symmetry becomes lost, and the crystal growth becomes impossible due to its hydration. Thus, ions can serve only as a source of fine droplets. The freezing point for the mixture of water and nitric acid is far below the freezing point of water (for nitric acid trihydrate, $\mathrm{T}_{\mathrm{NAT}}=192 \mathrm{~K}$ ). Perhaps for this reason, given the low threshold of supersaturation necessary for droplet growth in the mixture, in PSCs, the smaller supercooled drops are found in a mixture of water with nitric acid, whereas at the PSC boundary, where the concentration of $\mathrm{HNO}_{3}$ is low, we see fine, solid particles, apparently of ice [60].

Let us return to the estimations of the charge 
accumulation on the PSC boundary. On the basis of the analysis presented, it is possible to conclude that at the saturation rates near $100 \%$, the formation of the droplets with radii $\sim 1.2 \mathrm{~nm}$ (Fig. 9) begins. Their size may increase by a factor of 2 because of dissolution of a small amount of $\mathrm{HNO}_{3}$ inside their volume. For droplet sizes in the 1.2-2.4-nm range, the Langevin function varies in the interval 0.7-0.4, indicating a substantial loss of asymmetry of the water molecules in the droplet, which in turn results in a significant weakening of the external field by the water molecules, and the droplet mobility (with the ion inside it) decreases inside a cloud. If the field weakens by $\varepsilon=$ 80 times (where $\varepsilon$ is the dielectric permittivity of water) and the mobility changes because of an increase in the droplet size, the droplet velocity motion will decrease by more than two orders of magnitude.

In fact, such a droplet (with the ion inside) no longer participates in the electric current formation. The time for the formation of a droplet in the 1-2-nm size range is approximately $10^{-3}-10^{-2} \mathrm{~s}[26]$. From Eq. (8) one can easily estimate the equilibrium values of the ion concentration $n$ inside the aerosol layer. While $\mathrm{k} \sim 10^{2}-10^{3} 1 / \mathrm{s} \mathrm{n}$ will be of the order $\sim 5 \times 10^{-2}-5 \times 10^{-3}$ ion $/ \mathrm{cm}^{3}$. The substitution of those values into Eqs. (11) and (12) allows to estimate the quantity and concentration of the unipolar ions at the cloud boundaries. As one can see in the last line of the Table 3 the ion concentration at the aerosol layers boundaries can be equal or even exceed the background ion concentration in the layer of about $\sim 1 \mathrm{~km}$.

Thus the condensation processes of liquids on the ions may be accompanied by the significant decrease of ion concentration inside the aerosol layer and considerable growth of number of the unipolar ions at the aerosol layers boundaries.

Analysis of the measurements over Kiruna shows that the distribution of condensation nuclei is virtually identical to the character of the distribution of the ions that accumulate at the boundaries of the cloud and stick to the PSC droplets inside the cloud. An increase in the number of condensation nuclei increases the concentration of ions and vice versa, and with a decrease in the ion concentration, the concentration of condensation nuclei also decreases. Similar behaviour exhibited by ions and condensation nuclei suggests a correlation between them. Research has shown that this assumption is supported by theoretical and experimental investigation [61]. As mentioned above, it is possible that the ions contribute to the formation of condensation nuclei, which are manifested as small ice particles [60].

Thus, condensation nuclei can serve as a tracer for the presence of ions in the atmosphere. Of all charged particles, ions that constitute supercharged particles contribute most greatly to ozone depletion and to that of other compounds. It is very difficult to measure the ion composition in the stratosphere experimentally. However, condensation nuclei are measured in many airborne experiments.

\section{The Role of Ions in Stratospheric Photochemistry according to Experiments}

Analysis of a number of airborne experiments within the SOLVE (SAGE III Ozone Loss and Validation Experiment 1999-2000) experiments in the Arctic [62] and AAOE (Airborne Antarctic Ozone Experiment 1987) in the Antarctic [63] showed that the concentration of ozone and a number of other compounds in the stratosphere present a strong negative correlation with the concentration of condensation nuclei associated with ions.

This has been confirmed by measurements made using an ER-2 aircraft on 11 March 2000 [62] (Figs. 10 and 11). A depletion of the ozone was observed at this time, permitting an exploration of its link with the various stratospheric processes. A plane flew from Kiruna, located inside the polar vortex, towards the edge of the vortex, and then turned into higher latitudes. After this, the plane returned to Kiruna. The plane reached altitudes of $\sim 20 \mathrm{~km}$. At $\sim 40,000$ seconds, the plane descended to an altitude of $\sim 16 \mathrm{~km}$. 
CN

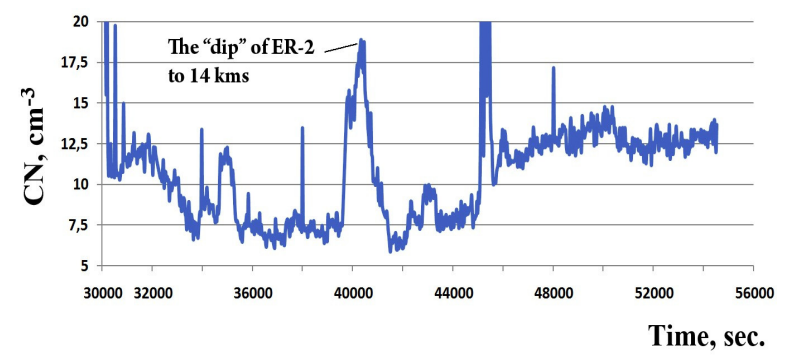

$1 / \mathrm{O}_{3}$

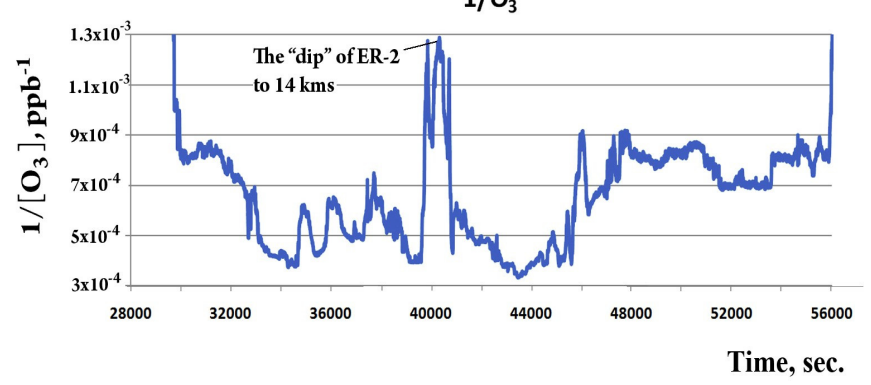

ClO

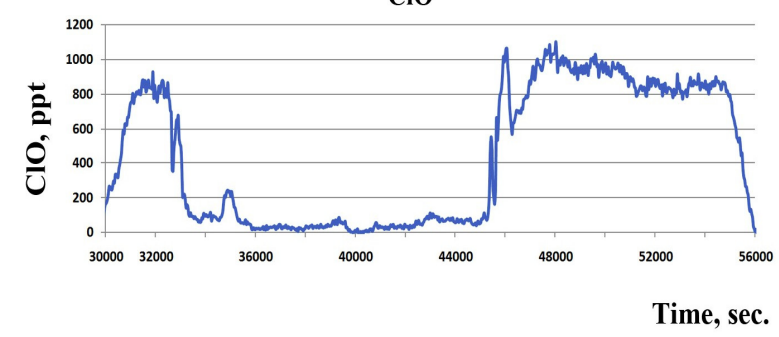

Aerosol total surface area

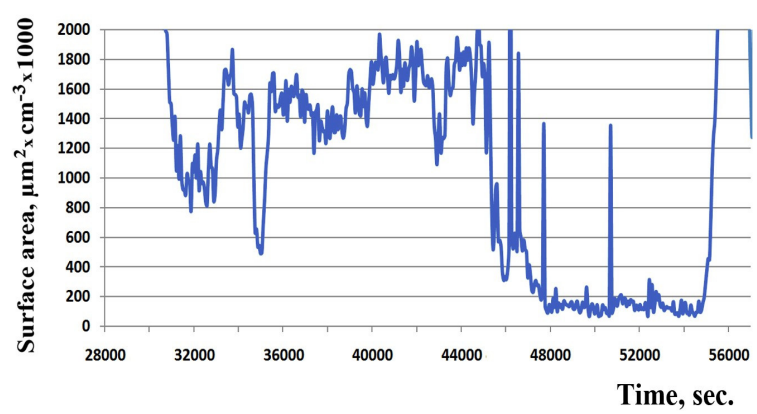

$\mathrm{N}_{2} \mathrm{O}$

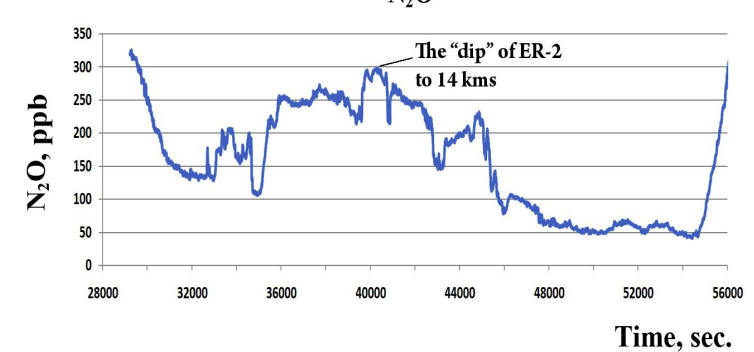

Concentration of molecules of air, $\mathrm{N}_{2}$

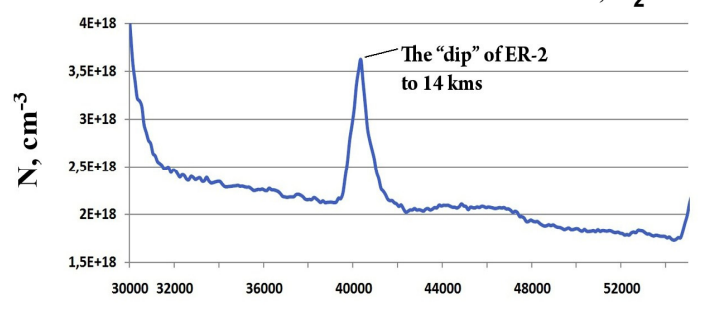

Time, sec.

Fig. 10 Airborne measurements in the Arctic on 11 March 2000 as part of the SOLVE experiment [62] (the left part of the figure is taken from our article [22]). Correlations of the inverse values of the mixing ratios of the ozone and condensation nuclei were observed even where there is no $\mathrm{ClO}$. This indicates that the main role in the destruction of $\mathrm{O}_{3}$ is played by ions. $\mathrm{N}_{2} \mathrm{O}$ is also destroyed on the ions: variations of $\mathrm{N}_{2} \mathrm{O}\left(\mathrm{O}_{3}\right)$ are associated with variations in the surface area of the aerosol (drops) due to the attachment of ions to the surface of drops and changes in the concentration of ions due to their hydration. The greatest destruction of $\mathrm{O}_{3}, \mathrm{~N}_{2} \mathrm{O}, \mathrm{CH}_{4}$ etc., was observed over the upper boundaries of PSCs $(\sim 31,000-33,000$ sec and $\sim 46,000-54,000 \mathrm{sec})$, where, due to the global electric circuit, the highest number of ions (condensation nuclei) accumulates.

During the greater period of the aeroplane's flight, the condensation nuclei were positively correlated with the inverse values of the ozone mixing ratio, including the flight segments where practically no $\mathrm{ClO}$ was found. The conclusion that chlorine oxide played a crucial role in the destruction of ozone was based on the observed negative correlation of ozone and chlorine monoxide (or on positive correlation of the chlorine monoxide and the inverse values of the ozone mixing ratio). This correlation was evident in the aircraft measurements on 11 March but only during certain segments of the flight.

Those observations indicate that alongside with the chlorine mechanism of the ozone destruction there is another mechanism of the ozone depletion on the charged particles. The share of each of the mentioned mechanisms contribution in the ozone destruction should be determined. The action of the ozone mechanism destruction which is related to the charged particles is vividly demonstrated at those passes of flight, where $\mathrm{ClO}$ is practically absent, especially during plane "dip". The $\mathrm{ClO}$ correlation with condensation nuclei 

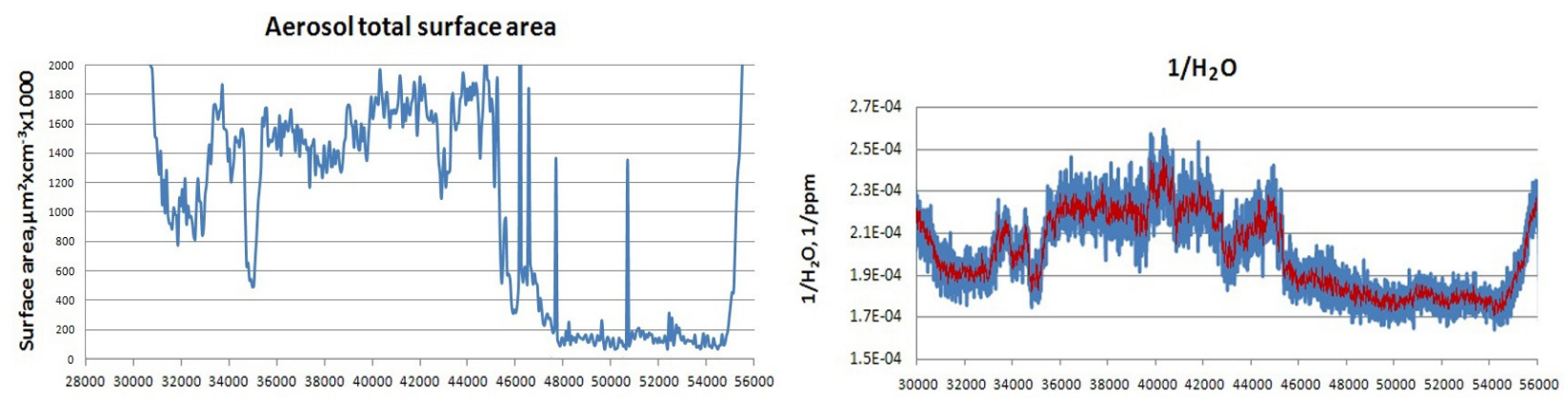

$\mathrm{CH}_{4}$
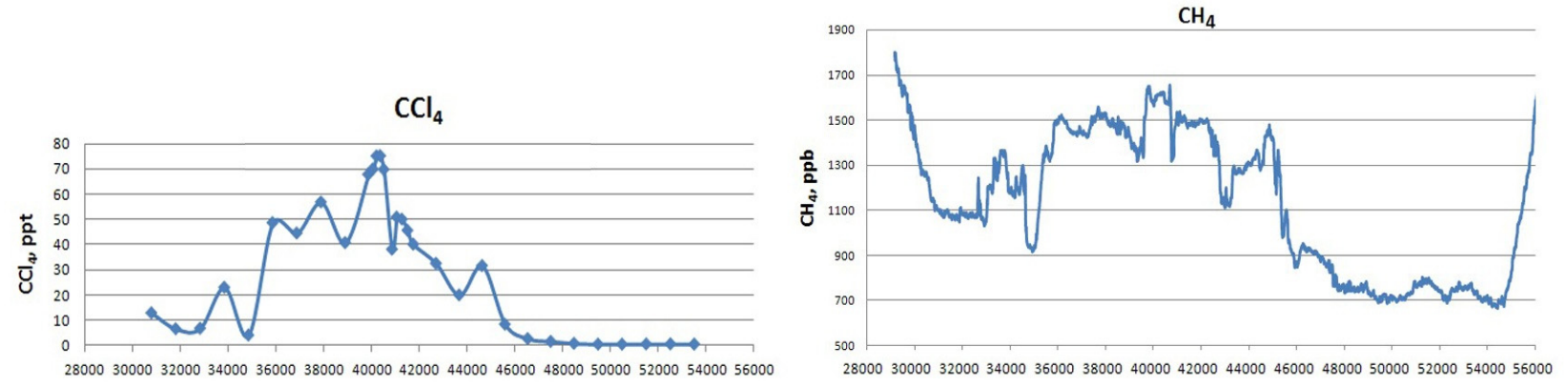

CFC11

CFC12
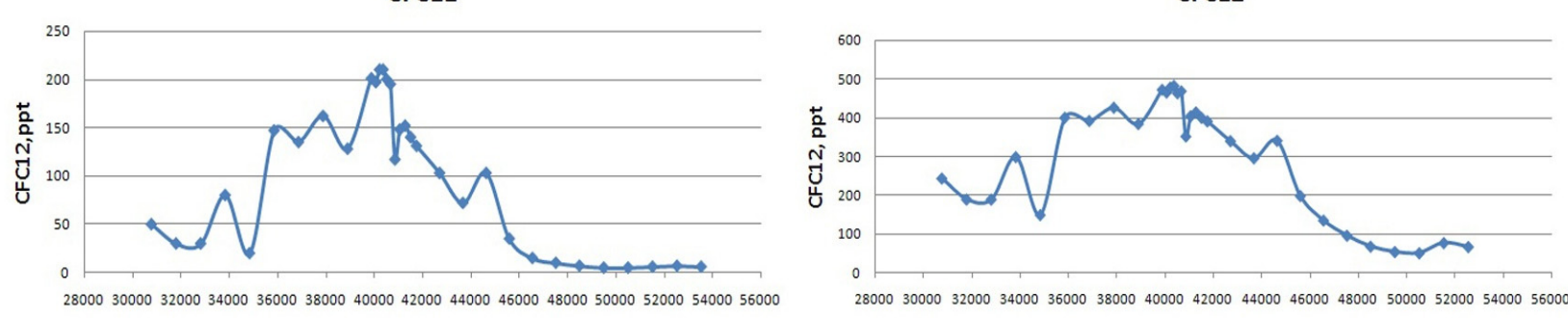

Time, sec.

Time, sec.

Fig. 11 Variations of the aerosol total surface area and mixing ratios of various compounds concentrations along the aeroplane flight path on March 11, 2000 in the SOLVE experiment [62] are shown. $\mathrm{CCl}_{4}, \mathrm{CH}_{4}$ and $\mathrm{CFCs}$ content variations are related to the ion variations, the concentrations of the latter ones depend on the aerosol fraction. The negative correlation between water vapor concentration and aerosol content is a consequence of a phase transition, that is a transformation of water vapour into droplets.

that means also with the charged particles is not coincidental. In our opinion the interaction of the chlorine reservoirs with the charged particles may bring to active chlorine release and $\mathrm{ClO}$ accumulation. This issue will be discussed below.

The irregularity in the measurement of the ozone profile observed along the flight path is associated with the breakdown of $\mathrm{O}_{3}$ at participation of ions (see Table 1), which are distributed unevenly due to their hydration as well as their adherence to PSC drops. The latter conclusion is confirmed by the negative correlation between the area of the PSC drops and the concentration of condensation nuclei (Figs. 10 and 12).
If the area of the PSC drops was high, the concentration of ions was low and less $\mathrm{O}_{3}$ destruction was evident, and vice versa. Recall that according to our estimates, charged PSC drops are not supercharged particles, and therefore, the destruction of ozone and other compounds on the surface of the drops does not occur.

Similar correlations along the flight path of the aircraft are observed between the concentration of condensation nuclei and the inverse values of the concentrations of such compounds as $\mathrm{N}_{2} \mathrm{O}, \mathrm{CH}_{4}$, CFC11 $\left(\mathrm{CFCl}_{3}\right), \mathrm{CFC} 12\left(\mathrm{CF}_{2} \mathrm{Cl}_{2}\right), \mathrm{CCl}_{4}$ etc. (Figs. 10 and 11). These compounds are considered to be 
chemically stable in the lower stratosphere, and the changes in their concentration are usually associated with atmospheric dynamics [64].

But in such case the concentration variations of the given compounds should be corresponded to air concentration variations (the mixing ratio must be constant). At the same time the mixing ratio of those compounds changes. One can see this in Figs. 10 and 11: a smooth air molecules concentration variations (except during the dip at $\sim 40,000 \mathrm{sec}$ ) are in a contrast with the uneven character change of the above mentioned compounds. That favor for photochemical effects which are not related to the atmospheric dynamics.

Our analysis shows that these compounds destruction in the lower stratosphere and that the cause of this decay is their interaction with ions. In our opinion the similar character of the above mentioned species destruction (Figs. 10 and 11) is a consequence of destruction of those species in the regime of "collision" (see Section 6 for more details). In this case a rate of destruction is determined mainly by the total effective area of the charged particles. Therefore even the compounds which greatly differ by the concentrations (e.g. $\mathrm{N}_{2} \mathrm{O}$ and $\mathrm{CCl}_{4}$ ) destruct similarly approximately with the same rate (the rate difference is about a factor of $2-3$ ).

One can see from Figs. 10, 11 and 12a that the regions where the most intensive destruction of $\mathrm{O}_{3}$, $\mathrm{N}_{2} \mathrm{O}, \mathrm{CH}_{4}$, etc. is observed are the coldest ones. One can infer from Figs. 10 and 11 that the highest nuclei concentration is observed in those areas, but the most unexpected result that there is also the lowest aerosol particle concentration (the plane flew near the cloud boundary, or above it).

The last fact does not correspond to the generally accepted view of the aerosol and PSC roles in the chlorine activation and the ozone destruction. Indeed, the greater the aerosol and PSCs area, the more intensive heterogenic reactions on the PSCs particles should be. Nevertheless, the aircraft observations in situ show, that is not the case. In the intermediary area between aerosol layers and over the aerosol and cloud layers surface the active chlorine accumulation and ozone depletion proceed more rapidly than inside the layers (Figs. 12b-d).

Such character of the ozone destruction and active chlorine accumulation is related to the ions and charged particles concentration rise take place in the area between aerosol and clouds layers. This conclusion is confirmed by the form of the condensation nuclei (ions) distribution given in Figs. 10 and 12e. We reckon that the main results of the global electric circuit work (the accumulation of ions) expose itself not only at the upper and lower cloud boundaries but also at the every local level, thus enhancing the presence of ions of both signs in the interspaces of the aerosol and clouds layers. Note that effect is enhanced in the daytime, because the electric currents in the stratosphere expose an essential diurnal range [46].

As far as relation between PSC area and ozone depletion is concerned, we confirm that it is surely present, and the enlargement of the PSC area brings to ozone concentration fall. There is no any contradiction with the discussed above. The PSCs presence are developed at low temperatures, but it is low temperatures and atmospheric dynamics are in our opinion the key processes for the ozone hole formation. As a rule, a more intensive vortex with a longer lifetime is attended with the greater coverage by PSC.

That is why the PSC area enlargement gives rise to the increase of ozone destruction rate, but this destruction takes place, first of all, above the upper boundaries of the clouds. There, in this region, both the enhanced ozone destruction on ions and enhanced decay chloric (halogenoid) reservoirs on ions (see below) take place. This effect causes the additional ozone destruction by means of the well-known catalytic cycles (see Section 6 and 7 for more details).

We have done similar conclusions for the Antarctic (Fig. 13). Analysis of airborne measurements of ozone 


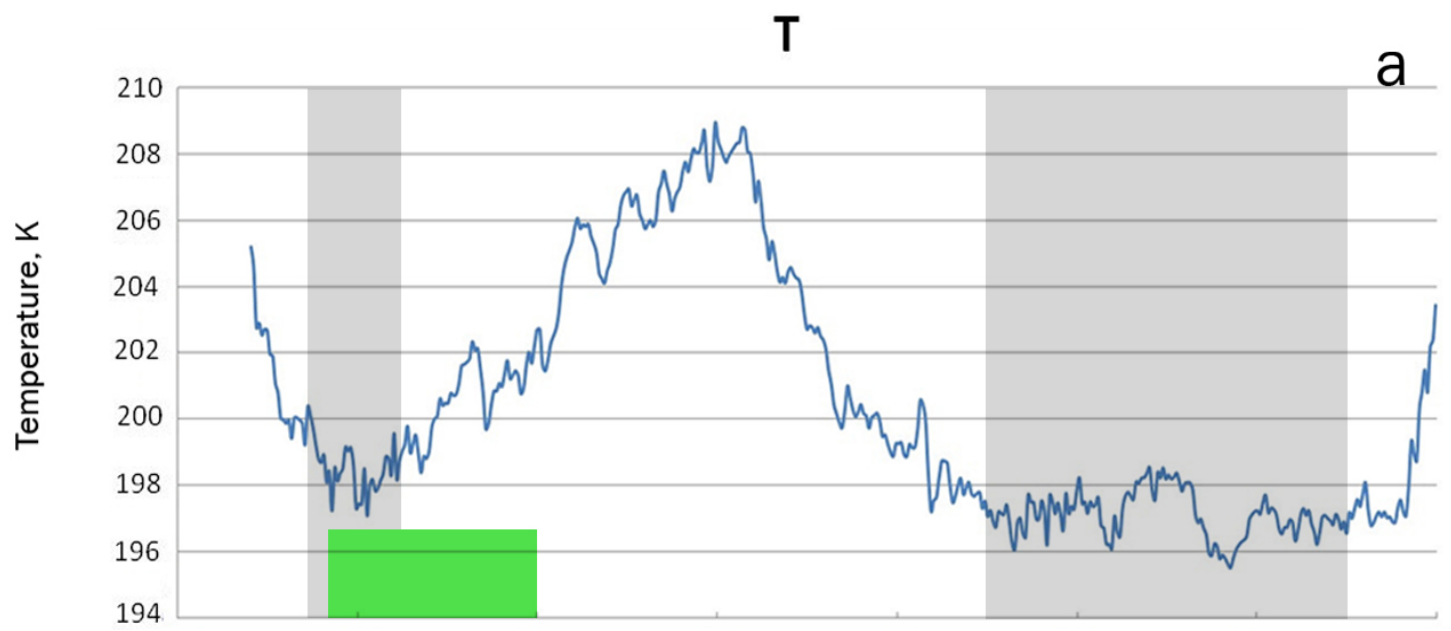

280003000032000340003600038000400004200044000460004800050000520005400056000

Time, sec.
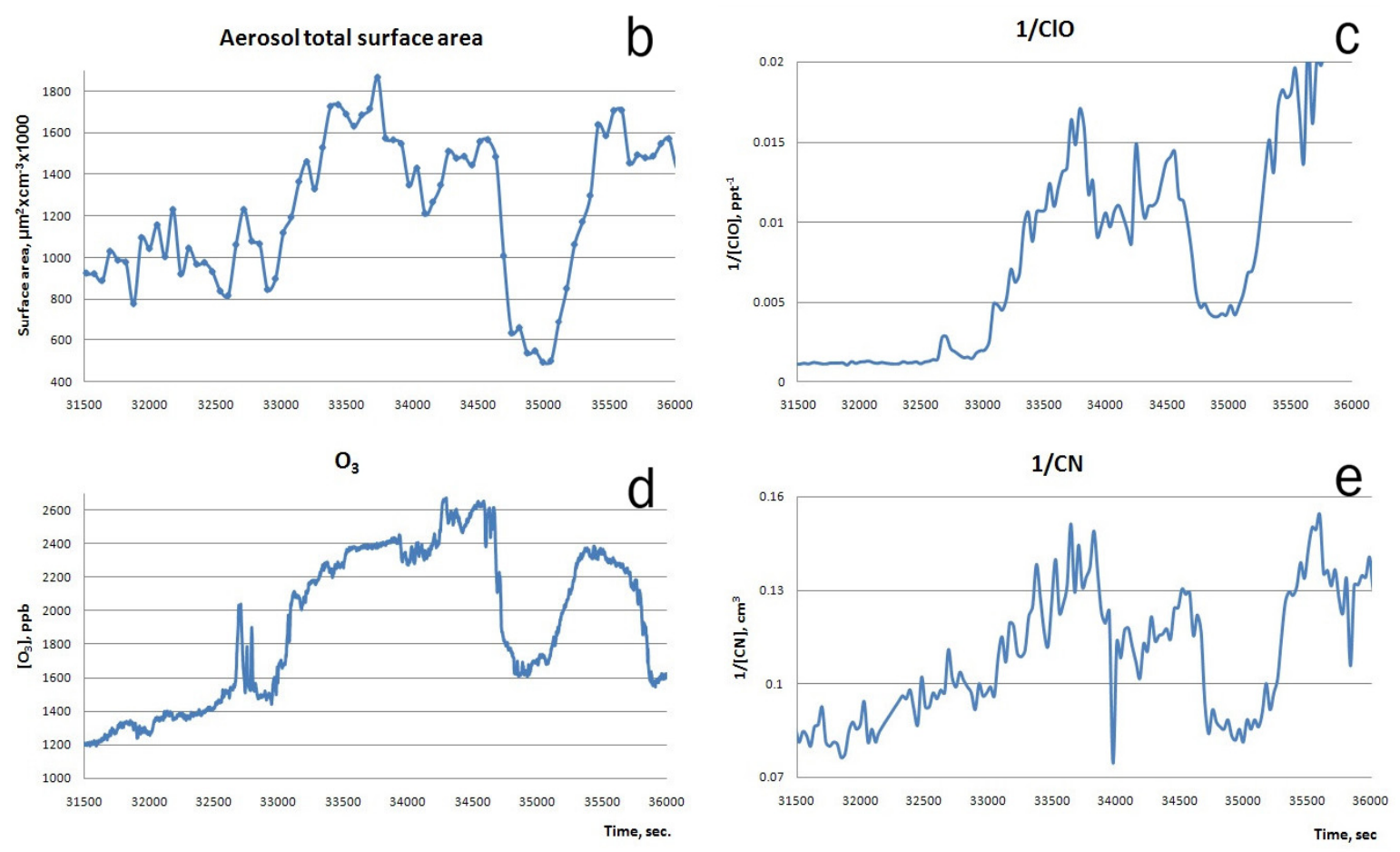

Fig. 12 The chlorine activation and ozone depletion take place not inside the aerosol layers but between them and over their boundary. In the upper figure part (a) the variation of temperature along the flight path on March 11, 2000 in the SOLVE experiment [62] is shown. The areas where $\mathrm{ClO}$ is accumulated extensively and the ozone concentration decreases are also shown (in grey). At the same time the aerosol concentration is decreased in those areas-which is caused, probably, with a descent of the upper cloud boundaries in the region of cold temperatures (note that the flight path was above the upper cloud boundary). The chlorine activation decreases and ozone concentration rises with the increasing of the aerosol surface, and vice versa. This effect is shown in detailed scale (b-d) at the short part of the plane flight (marked in green in the upper figure). At the same time the condensation nuclei (ions) are accumulated between aerosol layers and over the upper boundaries of the cloud layer (e), thus increasing the destruction of ozone and some other compounds in these areas. 


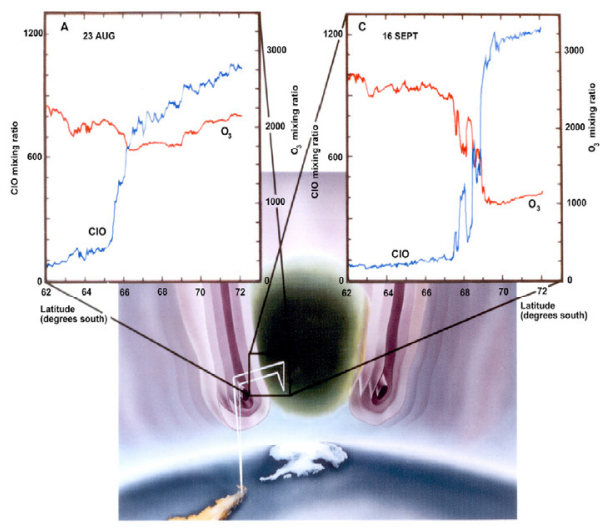

23 August, 1987

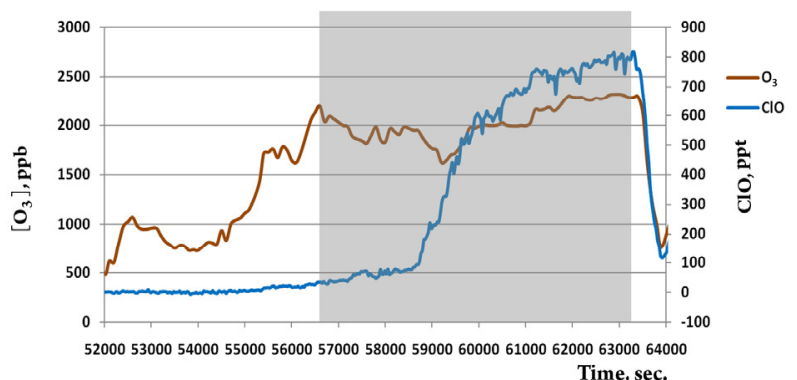

$\mathrm{O}_{3}$

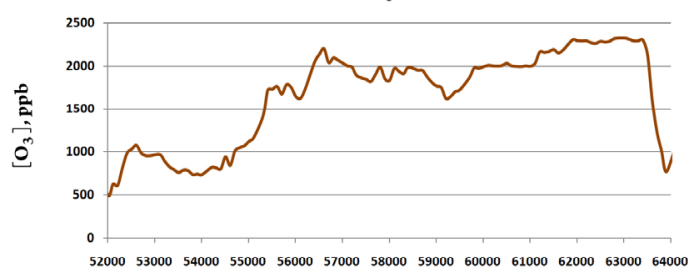

Time, sec.

$1 /(\mathrm{CNx} 10)$

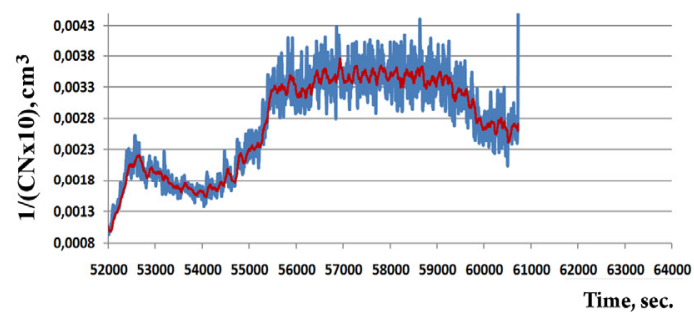

16 September, 1987
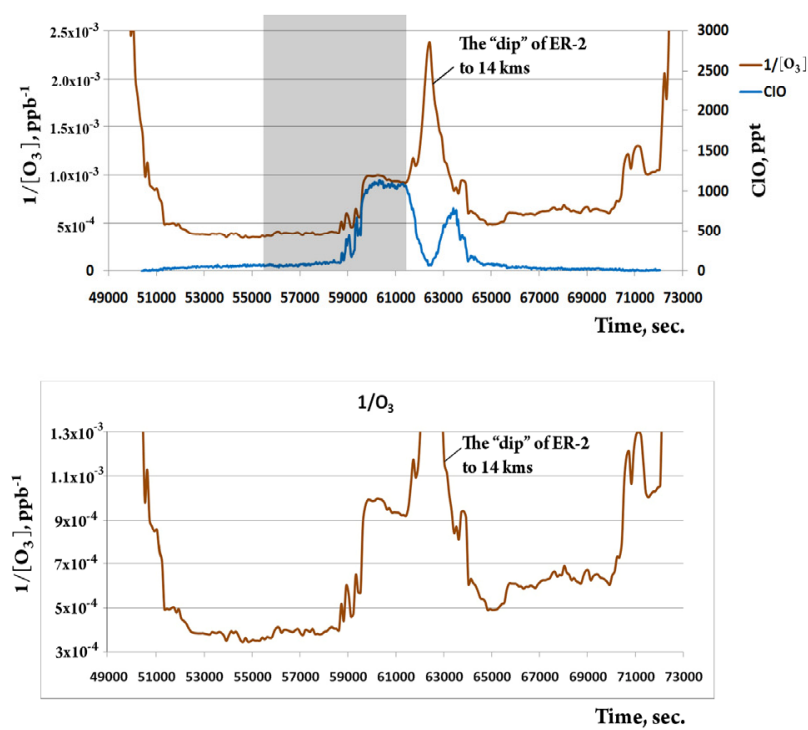

CNx10

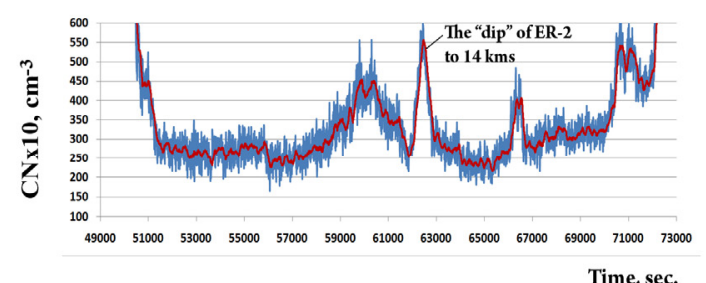

Fig. 13 Measurements made on an ER-2 aircraft on 23 August and 16 September 1987 in an AAOE experiment [63]: an illustration of measurements of ozone and chlorine monoxide in the Antarctic during the flight of the ER-2 aircraft into the vortex on 23 August and 16 September [65] (top); the measurement made on 23 August shows the mixing ratio of ozone (ppb), chlorine monoxide (ppt) and the inverse values of the concentration of condensation nuclei $\mathrm{CN}$ increased by 10-fold; measurements on 16 September show the values of the inverse ratio of a mixture of ozone, the mixing ratio of chlorine monoxide (ppt), and the concentration of condensation nuclei $\mathrm{CN}$ increased by 10 -fold. The results of measurements taken throughout the flight path of the aircraft (constructed on publicly available data) are shown in the lower part of Fig. 12. The shaded areas are flight segments, as indicated in the upper charts. 


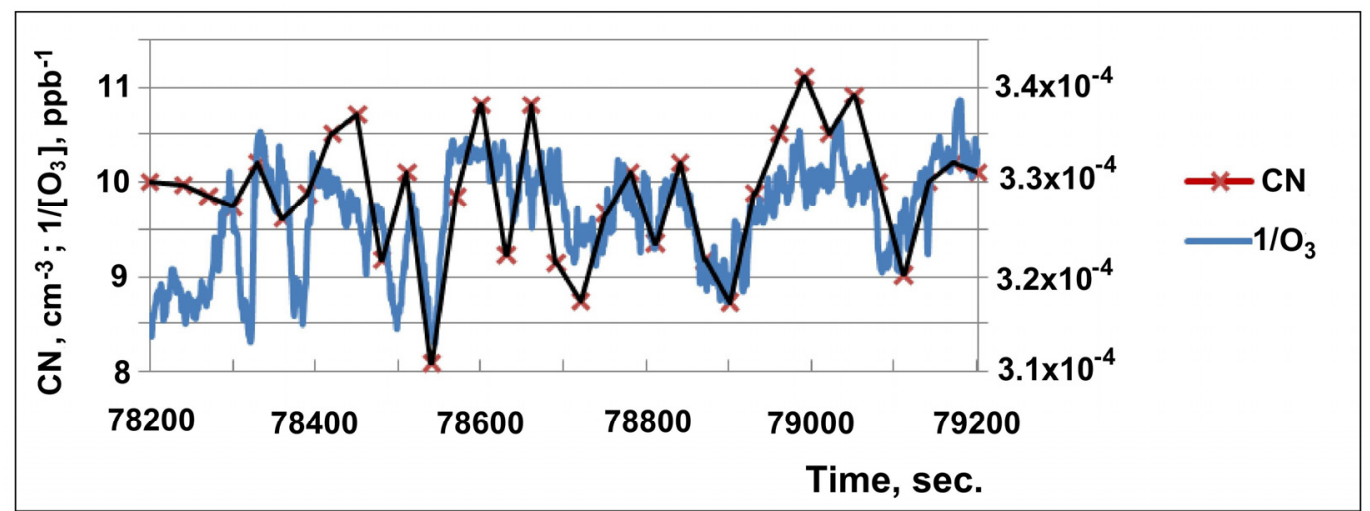

Fig. 14 Changes in inverse values of the ozone mixing ratio and concentrations of condensation nuclei along the part of the ER-2 flight path at night in the Arctic on 3 February 2000 as the part of the SOLVE experiment [62]. The altitude of the flight was approximately $19 \mathrm{~km}$.

and chlorine monoxide concentrations in the polar stratosphere for two days, 23 August and 16 September 1987 (as part of the AAOE experiment [63, 65]), showed that the negative correlation between ozone and chlorine monoxide was present only on 16 September in the early spring, and then only during a limited segment of the flight. At the same time, a negative correlation between the condensation nuclei and ozone to a varying degree was noted throughout the flight path in both airborne experiments.

Our analysis of measurements taken on 3 February 2000 during a night flight of an ER-2 aircraft as part of a SOLVE experiment [62] inside the polar vortex convinces us of the existence of the additional mechanism of ozone destruction (Fig. 14).

The zenith angle of the Sun throughout the flight was $\sim 114-115$ degrees. During this flight, the plane gradually gained altitude in stages. Fig. 14 shows the last part of the flight, corresponding to the maximum altitude of $\sim 19 \mathrm{~km}$. In this segment, the plane flew over or near the top of the aerosol layer. We have made this inference on the basis of the analysis of a change in the area of aerosol particles from 6-8 $\mu \mathrm{m}^{2} / \mathrm{cm}^{3}$ at the height of $14.5 \mathrm{~km}$ at the beginning of the flight to $\sim 1-2 \mu \mathrm{m}^{2} / \mathrm{cm}^{3}$ on the specified site at the altitude of approximately $19 \mathrm{~km}$. This provides the best example of the negative correlation between ozone and the number of condensation nuclei.

Negative correlation with chlorine monoxide is also present (not shown here). The reason for the ozone variation at night cannot be the $\mathrm{ClO}$ dimer cycle because this requires the solar radiation. However, the presence of ions in the lower stratosphere can explain the variations in the ozone. Note please as it was marked earlier that there are diurnal variations of currents [46] responsible for the accumulation of charges on the upper boundary of the cloud. At night, these currents are very weak, so that even above the clouds, the destruction of the ozone is minimal.

Thus the presented analysis confirms the existence of the source of the ozone destruction which is independent from $\mathrm{ClO}$ and is related to the charged particles.

\section{Photochemistry of the Polar Stratosphere with the Participation of Charged Particles}

The previous analysis of the experimental results confirms our hypothesis about the role of the charged particles in the polar stratosphere. However, questions about the details of the behaviour of photochemical reactions remain. First, what is the cause of the ozone destruction? Initially, we thought that the ozone molecule was broken down into charged particles, but because its decay products are the symmetric molecule $\mathrm{O}_{2}$ and the atom $\mathrm{O}$, both of which have a zero dipole moment. After the destruction of the ozone molecule, they should be removed from the surface of a charged particle into space where the 
ozone molecule has been recovered:

$$
\begin{aligned}
& \mathrm{O}_{3}(\mathrm{v}) \rightarrow \mathrm{O}_{3}(\mathrm{~s}) ; \mathrm{O}_{3}(\mathrm{~s})+\mathrm{M}(\mathrm{s}) \rightarrow \\
& \mathrm{O}_{2}(\mathrm{~s})+\mathrm{O}(\mathrm{s})+\mathrm{M}(\mathrm{s})+1.4 \mathrm{eV}, \\
& \mathrm{O}_{2}(\mathrm{~s}) \rightarrow \mathrm{O}_{2}(\mathrm{v}) ; \mathrm{O}(\mathrm{s}) \rightarrow \mathrm{O}(\mathrm{v}) \text { and } \\
& \mathrm{O}_{2}(\mathrm{v})+\mathrm{O}(\mathrm{v})+\mathrm{M}(\mathrm{v}) \rightarrow \mathrm{O}_{3}(\mathrm{v})+\mathrm{M}(\mathrm{v})
\end{aligned}
$$

In the above chemical reactions, $\mathrm{s}$ and $\mathrm{v}$ denote the locations of molecules: ambient space is $\mathrm{v}$ (volume), and the surface of a charged particle is denoted by $\mathrm{s}$ (surface).

Based on this reaction scheme, no depletion of the ozone in the stratosphere should be observed. Obviously, something has been overlooked in our analysis. A closer look at the above reaction scheme is warranted.

Emerging from the effective surface of the ion molecules into ambient space $\mathrm{O}_{2}(\mathrm{~s})$ and $\mathrm{O}(\mathrm{s})$ occur mainly via the collision of these molecules with the molecules surrounding the charged particle $\mathrm{H}_{2} \mathrm{O}(\mathrm{s})$, and if the molecule of $\mathrm{O}_{2}(\mathrm{~s})$ does not react upon a collision with the molecule $\mathrm{H}_{2} \mathrm{O}(\mathrm{s})$, then the oxygen atom $\mathrm{O}(\mathrm{s})$ can interact with the water molecules by means of the following reaction:

$$
\mathrm{O}(\mathrm{s})+\mathrm{H}_{2} \mathrm{O}(\mathrm{s}) \rightarrow 2 \mathrm{OH}(\mathrm{s})
$$

According to the database of the National Institute of Standards and Technology [66], the response threshold of the reaction shown in Eq. (18) is $\sim 0.7 \mathrm{eV}$. At the same time, the potential energy of the water molecules on the surface of the ion $\mathrm{H}_{3} \mathrm{O}^{+}$(the most widespread positive ion in the stratosphere) is $\sim 0.8$ $\mathrm{eV}$ and might be involved into this reaction. Furthermore, the energy of $\sim 1.4 \mathrm{eV}$ (16) released during the ozone decay might be partially involved in this reaction. We must also consider the tremendous effective density of water molecules on the surface.

These estimates imply that on the effective surface of the ion in this reaction, the energy of $\sim 0.8-2.2 \mathrm{eV}$ may be activated, which is larger than the threshold of $\sim 0.7 \mathrm{eV}$ for this reaction. Thus, ozone is a powerful source of the $\mathrm{OH}$ molecules on the surface of the charged particles, and the concentration of ozone $(\sim 1$ $\times 10^{12}$ molecules $\left./ \mathrm{cm}^{3}\right)$ in the stratosphere exceeds the concentration of chlorine and nitrogen compounds and is comparable to the concentration of water molecules that are necessary for the hydroxyl radical reproduction.

The hydroxyl radical molecule lingers for some time on the surface of the ion because the lifetime of this molecule on the surface is comparable with the lifetime of a water molecule because of the high dipole moment of $\mathrm{OH}$, which is $\sim 1.65 \mathrm{D}$ (for the water molecule, it is $\sim 1.85 \mathrm{D})$. Given this fact, as well as the high concentration of ozone in the stratosphere which iteratively exceeds those ones of the chlorine, nitrogen and other compounds, we concluded that the series of compounds can react on the effective ion surface with $\mathrm{OH}$, which is formed as a result of the above given reactions. Obviously, in this process, the ozone molecule serving as the source of hydroxyl radical is irreversibly destroyed.

Table 4 shows some of the reactions involving $\mathrm{OH}$, which we believe to occur on the surface of the supercharged particles. The coefficients of these reactions $\left(\mathrm{K}_{\text {space }}\right)$ and activation energies of the indicated compounds $\left(\mathrm{E}_{\text {space }}\right)$ in space were evaluated according to the databases of chemical reactions of the JPL [30] and of the NIST [66]. Note that the potential energy of $\mathrm{OH}$ on the effective ion surface of $\mathrm{H}_{3} \mathrm{O}^{+}$is $\sim 0.7 \mathrm{eV}$. This exceeds the threshold value of all of the reactions listed in Table 4.

The activation energy $E_{\text {surface }}$ on the surface of the supercharged particle with the participation of the hydroxyl radical can be estimated as $\mathrm{E}_{\text {surface }}=\mathrm{E}_{\text {space }}$ 0.7. The activation energies $\mathrm{E}_{\text {surface }}$ of some compounds are also given in Table 4. It should be pointed out that by no means all compounds, which can interact with the hydroxyl radical $\mathrm{OH}$ on the charged particle surface are presented in Table 4.

Please note the first two reactions in Table 4 which lead to the accumulation of the active chlorine in the stratosphere. In our opinion the active chlorine extraction in the presence of the charged particles takes place from both chlorine reservoirs $\mathrm{HCl}$ and 
Table 4 The reaction coefficients in the space, activation energies of some compounds in the space $E_{\text {space }}$ and on the surface $E_{\text {surface }}$ of supercharged particles for $T=190 \mathrm{~K}$.

\begin{tabular}{lll}
\hline Reactions & Reaction coefficient, & Activation energy, eV \\
& $\mathrm{K}_{\text {space }}, \mathrm{cm}^{3}$ molecule $\mathrm{s}^{-1}$ & $\mathrm{E}_{\text {space }} / \mathrm{E}_{\text {surface }}$ \\
\hline $\mathrm{OH}+\mathrm{HCl} \rightarrow \mathrm{Cl}+\mathrm{H}_{2} \mathrm{O}$ & $2.6 \times 10^{-12}$ & $0.03 /-0.67$ \\
$\mathrm{OH}+\mathrm{ClONO} \mathrm{H}_{2} \rightarrow \mathrm{ClO}+\mathrm{HNO}_{3}$ & $1.2 \times 10^{-12}$ & $0.03 /-0.67$ \\
$\mathrm{OH}+\mathrm{HOCl} \rightarrow \mathrm{ClO}+\mathrm{H}_{2} \mathrm{O}$ & $3.10 \times 10^{-12}$ & $0.05 /-0.65$ \\
$\mathrm{OH}+\mathrm{ClO} \rightarrow \mathrm{Cl}+\mathrm{HO}_{2}$ & $7.4 \times 10^{-12}$ & $-0.02 /-0.72$ \\
$\mathrm{OH}+\mathrm{ClO} \rightarrow \mathrm{HCl}+\mathrm{O}_{2}$ & $6.0 \times 10^{-13}$ & $-0.02 /-0.72$ \\
$\mathrm{OH}+\mathrm{CH}_{4} \rightarrow \mathrm{CH}_{3}+\mathrm{H}_{2} \mathrm{O}$ & $2.45 \times 10^{-12}$ & $0.15 /-0.65$ \\
$\mathrm{OH}+\mathrm{N}_{2} \mathrm{O} \rightarrow \mathrm{HO}_{2}+\mathrm{N}_{2}$ & $1.4 \times 10^{-11}-3.8 \times 10^{-17}$ & $0.4 /-0.3$ \\
$\mathrm{OH}+\mathrm{CFCl}_{3}(\mathrm{CFC}-11) \rightarrow$ products $\left(\mathrm{CCl}{ }_{2} \mathrm{~F}+\mathrm{HOCl}\right)$ & $1.0 \times 10^{-12}$ & $>0.32 /-0.38$ \\
$\mathrm{OH}+\mathrm{CF}_{2} \mathrm{Cl}_{2}(\mathrm{CFC}-12) \rightarrow$ products $\left(\mathrm{CClF} \mathrm{F}_{2}+\mathrm{HOCl}\right)$ & $1.0 \times 10^{-12}$ & $>0.31 /-0.39$ \\
$\mathrm{OH}+\mathrm{CCl}_{4} \rightarrow$ products $\left(\mathrm{CCl}_{3}+\mathrm{HOCl}\right)$ & $1.0 \times 10^{-12}$ & $>0.20 /-0.50$ \\
\hline
\end{tabular}

$\mathrm{ClONO}_{2}$ separately and not as a result of the interaction of those reservoirs [21, 22]. The charged particles promote the active chlorine accumulation (see Figs. 10 and 12) and ozone destruction by means of the $\mathrm{ClO}$ dimer cycle together with direct ozone destruction on the charged particles.

We wish to illustrate the possible passing of the above-mentioned processes and how the chemical reactions on the charged particles surface and in free space are interrelated.

The interaction of the main chlorine reservoirs with the hydroxyl radical molecules $\mathrm{OH}$ on the charged particles surface gives rise to the following reactions:

$$
\begin{gathered}
\mathrm{OH}(\mathrm{s})+\mathrm{HCl}(\mathrm{s}) \rightarrow \mathrm{Cl}(\mathrm{v})+\mathrm{H}_{2} \mathrm{O}(\mathrm{s}) \\
\mathrm{OH}(\mathrm{s})+\mathrm{ClONO}_{2}(\mathrm{~s}) \rightarrow \mathrm{ClO}(\mathrm{s})+\mathrm{HNO}_{3}(\mathrm{~s})
\end{gathered}
$$

The chlorine atom $\mathrm{Cl}$ which is produced in reaction (19) is released in ambient space because it possesses the zero dipole moment. The molecules, produced by the reaction (20) have the large dipole moments and remain at the surface, but they can also interact with hydroxyl radical on the surface. Such interaction results in releasing of the chlorine atom out of $\mathrm{ClO}$ and injection of the former in ambient space:

$$
\mathrm{OH}(\mathrm{s})+\mathrm{ClO}(\mathrm{s}) \rightarrow \mathrm{Cl}(\mathrm{v})+\mathrm{HO}_{2}(\mathrm{~s})
$$

Note, that the reaction of $\mathrm{HCl}$ formation can also pass on the surface:

$$
\mathrm{OH}(\mathrm{s})+\mathrm{ClO}(\mathrm{s}) \rightarrow \mathrm{HCl}(\mathrm{s})+\mathrm{O}_{2}(\mathrm{v})
$$

However, the large dipole moment of the $\mathrm{HCl}$ molecule do not allow it to be released into space.
Therefore the removal of this molecule from the surface is possible only as a result of reaction (19) with escaping of the active chlorine into space:

$$
\mathrm{HCl}(\mathrm{s})+\mathrm{OH}(\mathrm{s}) \rightarrow \mathrm{Cl}(\mathrm{v})+\mathrm{H}_{2} \mathrm{O}(\mathrm{s})
$$

Thus chlorine atoms are released out of the chlorine reservoirs, and then, in ambient space, are involved into the $\mathrm{ClO}$ dimer cycle:

$$
\begin{array}{r}
\mathrm{Cl}+\mathrm{O}_{3} \rightarrow \mathrm{ClO}+\mathrm{O}_{2} ; \mathrm{ClO}+\mathrm{ClO} \rightarrow \mathrm{Cl}_{2} \mathrm{O}_{2} ; \\
\mathrm{Cl}_{2} \mathrm{O}_{2}+\mathrm{hv} \rightarrow 2 \mathrm{Cl}+\mathrm{O}_{2} \text { and so on. }
\end{array}
$$

Here hv is the solar radiation.

The reaction (22) passes in ambient space also:

$$
\mathrm{OH}(\mathrm{v})+\mathrm{ClO}(\mathrm{v}) \rightarrow \mathrm{HCl}(\mathrm{v})+\mathrm{O}_{2}(\mathrm{v})
$$

While the Sun elevation angle increases and $\mathrm{OH}$ concentration rises in space the $\mathrm{HCl}$ accumulations take place (the recovery stage). In the absence of spatial $\mathrm{OH}$ only reaction of the $\mathrm{HCl}$ decomposition passes with the yield of $\mathrm{Cl}$ (reaction 19).

It is worth to note, that the active chlorine accumulation as well as a set of other processes would be impossible without processes of desorption and filtration of molecules on the surface depending on the dipole moment.

In Ref. [21] some other possible reactions with the charged particles participations are considered.

Table 4 also shows that in the polar stratosphere, not only the ozone hole can be formed but also a methane hole, nitrous oxide hole, etc.

According to Ref. [21] such species as HOONO and $\mathrm{HNO}_{4}$ also can be the source of hydroxyl radical 
$\mathrm{OH}$ in the stratosphere since they can easily dissociate on the surface of the charged particles (see Table 1). The example of such dissociation of HOONO is given below.

The principles of renewing the ion environment and the reaction behaviour to its effective surface are shown in Fig. 15. The figure shows that the environment of the core ion $\mathrm{H}_{3} \mathrm{O}^{+}$is not constant and can be altered by other molecules, primarily molecules that have large dipole moments. In this regard, the rotation of water molecules is also possible. A water molecule undergoing a dipole interaction with an ion can acquire a kinetic energy $\sim 0.8 \mathrm{eV}$, similar to the potential energy of another sticky molecule of $\mathrm{H}_{2} \mathrm{O}$. As a result of such collision, one incident molecule takes the place of another that had become attached, pushing it beyond the "sticking" radius (the distance at which the potential energy of a molecule is equal to its kinetic thermal energy).

If a molecule with a small dipole moment (NO, $\mathrm{NO}_{2}$, etc.) and a correspondingly low binding energy comes into the proximity of the ion and bonds with it, it is ejected into space, encountering a water molecule with even a smaller kinetic energy. At the same time, a molecule with a large dipole moment, such as $\mathrm{OH}$, is not easily ejected from the environment of the ion because only very energetic water molecules of $\mathrm{H}_{2} \mathrm{O}$ can cause this to occur. This means that the lifetime of a $\mathrm{NO}_{2}$ molecule is significantly shorter than the lifetime of $\mathrm{OH}$ molecule on the effective surface of the ion.

Fig. 15 also demonstrates the principle of the chemical reactions on the effective surface of an ion. The molecule HOONO (the isomer of $\mathrm{HNO}_{3}$, [67]) has a sufficiently large dipole moment $(\mathrm{p} \sim 1.07 \mathrm{D})$ and can appear on the effective surface of an ion. The energy required for the dissipation of this molecule $\mathrm{E}_{\mathrm{act}}$. is $\sim 0.9 \mathrm{eV}$. As demonstrated in the figure, this energy is fully achievable by a collision with the $\mathrm{H}_{2} \mathrm{O}$ molecules in the ion environment.

The decay products behave differently. The $\mathrm{NO}_{2}$ molecule is ejected into ambient space, whereas the $\mathrm{OH}$ molecule remains on the surface and can react with a molecule trapped on the surface, such as a
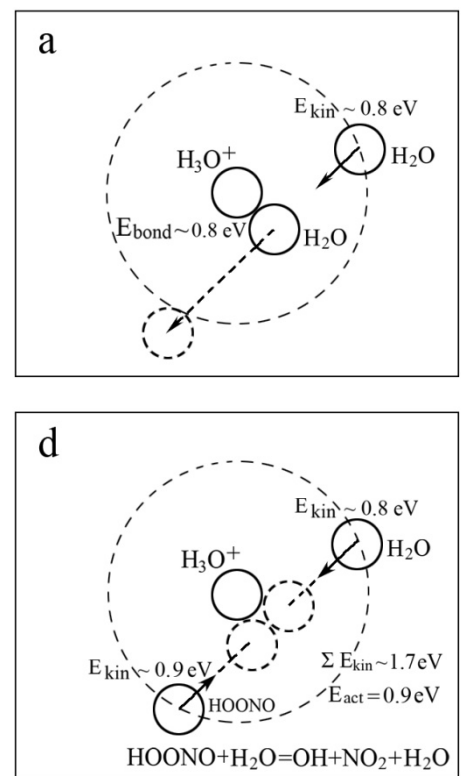
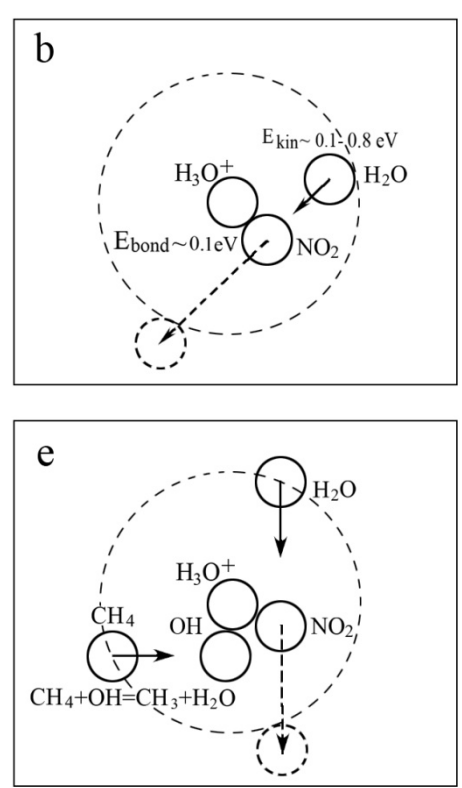
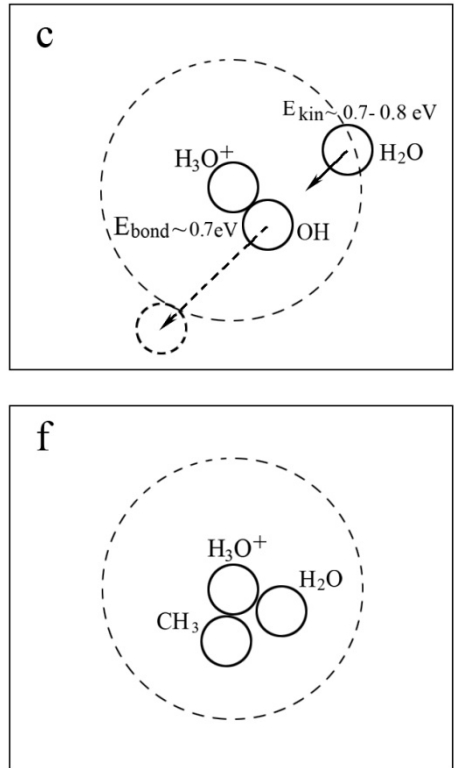

Fig.15 (a) The principle of the renewal of the environment of the core ion $\mathrm{H}_{3} \mathrm{O}^{+}$, containing water molecules. (b-c)- show that the molecules $\mathrm{NO}_{2}$ and $\mathrm{OH}$ have significantly different lifetimes on the surface because the range of energies of the $\mathrm{H}_{2} \mathrm{O}$ molecules, which can push molecules beyond the adhesion radius, is significantly different for these molecules. (d-f)-examples of chemical reactions on the ion surface (see text); the dashed circle indicates the adhesion radius of the water molecules. 
methane molecule. The $\mathrm{OH}$ molecule can also connect with $\mathrm{HCl}$ и $\mathrm{ClONO}_{2}$ molecules and extract their active chlorine.

In our photochemistry model, we show that with the participation of charged particles [21], the majority of the chemical compounds shown in Table 1 can be degraded on the surface of the supercharged particles in the regime of "collision". In this case, the lifetime of the chemical compounds can be estimated using Eq. (10):

$$
t=(P S v n / 4)^{-1}
$$

where $\mathrm{P}$ is the probability of the molecule sticking to the ion, $S$ is the effective surface of the ion, $n$ is the concentration of ions, and $\mathrm{v}$ is the average velocity of the molecules.

Based on these estimates and at the stratospheric ion concentration of $(1-5) \times 10^{3}$ ions $/ \mathrm{cm}^{3}$ and the ion effective radius equal to the adhesion radius [21] $\sim 10$ $\AA$ for $\mathrm{H}_{3} \mathrm{O}^{+}$), it is possible to evaluate the lower lifetime limit for the chemical compounds in the process of their collisions with ions. In such a case for $\mathrm{P}=1$, the lifetime of the compounds in Tables 1 and 4 varies from 5 days to 5 months. Similar calculations show that the lifetime of ozone changes from 10 days to 2 months at the specified variations of the ionic concentration. The corresponding rate of ozone depletion can change $\sim\left(2.4 \times 10^{-7}-1.2 \times 10^{-6}\right) 1 / \mathrm{s}$.

Thus the ozone destruction mechanism on the charged particles allows to explain the ozone depletion formation in the Polar Regions without involving the chlorine mechanism. Therewith the ozone destruction mechanism on the charged particles is considered as more universal as the chlorine one, because it works at all latitudes and altitudes, including those ones where $\mathrm{ClO}$ is absent or its concentration low. It seems quite natural the enhancement of this mechanism in the polar regions, where number of the charged particles is increased in comparison with the midlatitudes as the result of the effect of the cosmic rays [10]. In the presence of the charged particles a number of molecules together with ozone undergo to destruction processes (see Table 4, Figs. 10 and 11). The chlorine theory is not able to explain the destruction of other species together with ozone. At the same time in the presence of the charged particles the accumulation of the active chlorine takes place which can enforce the direct ozone destruction on the charged particles (see Eqs. 19-23). It is possible that contributions of both chlorine mechanism and that one of the ozone destruction on by the charged particles in the lower stratosphere are comparable with each other. In both mechanisms the charged particles distribution and stratospheric dynamics are the decisive factors in the ozone depletion formation.

\section{Ozone Depletion Formation in the Polar Stratosphere with Charged Particles Participation}

Our analysis of the polar experiments $[35,36,40$, $41,46,62-65]$ show that air descend inside the polar vortex results in involving ozone and some other compounds into altitudinal range with the enhances concentration of the charge particles, on which the ozone and other compounds destruction takes place. It is important to emphasize that together with the ozone destruction the chlorine-containing species: $\mathrm{HCl}$ and $\mathrm{ClONO}_{2}$ are also undergone to destruction. This process results in the active chlorine release and putting into work the $\mathrm{ClO}$ dimer cycle, which also causes the ozone destruction, Eqs. (19-24).

The presence of the charged particles in the polar stratosphere affects the ozone content by decreasing its concentration especially at those altitudes where the enlarged concentration of the charged particles is observed. As a rule, it is detected on the clouds and aerosol layers boundaries in the stratosphere and upper troposphere. The highest concentration of the charged particles corresponds to the upper cloud boundaries, and at the same time there are the local maxima present, which correspond to boundaries of every cloud layer (Figs. 4, 10, 11 and 12). 
In our opinion, such character of the charged particles distribution can be accounted for not only by the increase of ions of both signs outside aerosol layers (Fig. 7), but also as a consequence of the global electric circuit work, which results in accumulation of the unipolar (of one sign) charges on the clouds and aerosol layers boundaries; these charges are mainly due to ions, the positive charge being concentrated on the upper boundary, and the negative charge on the lower one (Fig. 8).

As it was marked earlier in a daytime the electric currents in the polar stratosphere, and that means also the unipolar charge accumulation over clouds can grow up a few times over consequent values in nighttime (three time according to Ref. [46]). Therefore the ozone and other compounds destruction rates increase in the springtime and continue to grow with the Sun elevation angle rising. This is confirmed by our analysis of data obtained in the ER-2 nighttime flight, when only insignificant variations of ozone concentrations are observed inside the polar vortex as well as the lower nighttime concentrations of condensation nuclei in comparison with daytime ones over the upper cloud boundaries (see Fig. 10, $\sim 46,000-54,000$ sec. and Fig. 14).

It is necessary to mention also a sufficient difference between condensation nuclei (ions) concentrations in Arctic and Antarctic. The comparison between Figs. 10 and 13 shows that along all over trajectory of ER-2 plane flight the condensation nuclei concentrations in Antarctic exceed those ones in Arctic. This difference is particularly evident above the upper PSC boundaries where the condensation nuclei concentrations in Antarctic can exceed those ones in Arctic by a factor of an order of magnitude (Fig. 4c). This factor affects directly the ozone and chlorine reservoirs destruction rate, and, in our opinion, may be one of the causes of the formation of the "real" ozone holes in Antarctic, while in Arctic mainly mini-holes are observed.

Our analysis showed that the main pretender for the ozone and other compounds destruction is a positive ion $\mathrm{H}_{3} \mathrm{O}^{+}\left(\mathrm{H}_{2} \mathrm{O}\right)_{n}$ with a small number of ligands $(\mathrm{n}<$ $10)$.

The analysis of the balloon measurements in Kiruna shows that the PSC upper boundary over Kiruna is at the altitude of $\sim 22-23 \mathrm{~km}$, just a little bit higher than this level a small ozone depletion is formed (Figs. 4a and $4 \mathrm{~b}$ ). Our analysis of ER-2 flights in the polar stratosphere shows that the increased condensation nuclei concentration, and, consequently, the ionic concentration exists at the altitudes above $\sim 17-20 \mathrm{~km}$ in the polar vortex (Figs.10 and 16b).

According to satellite observations [68] the upper PSC boundary can vary from $\sim 10-12 \mathrm{~km}$ to $25 \mathrm{~km}$, being sufficiently lower inside the vortex [68]. Kent et al. [69] showed that the Arctic vortex acts as a semi-porous barrier to transport above $\sim 14 \mathrm{~km}$, i.e., intra-vortical air is relatively isolated from lower latitude air masses which contain higher aerosol concentrations.

Basing on those results we make a conclusion that ozone and some other compounds can be destructed in a whole altitudinal range where PSC and aerosol layers are present. However this destruction rates are very different inside the cloud and its circumference, as well as for aerosol layers. Thus very "uneven character" of the ozone distribution both by altitude and in ambient space is formed (Figs. 4, 10, 12 and 13). The horizontal advection smoothes ozone concentration inside the vortex on given altitude layer almost without changing the altitudinal ozone distribution. It is altitude and depth of clouds which are efficient factors which ozone destruction rate depends on.

Illustration for those conclusions is given in Fig. 16a. The air descend inside the vortex is shown in the top by the vertical arrows. Ozone and other compounds together with air reach those altitudes where the increased content of the charged particles which are distributed rather non-uniformly. The reasons of such non-uniform charge distribution as it was mentioned above are a presence of PSC (shaded area) and the global electric circuit work. 
On account of the non-uniform charge height distribution it is difficult to estimate the integral ozone destruction rate on the charged particles inside the vortex, as well as to determine ozone concentration variations from winter to spring seasons taking into account changes in cloudiness, cloud depth, and cloud altitude. That is also true for other compounds including chlorine reservoirs. One must also take into account horizontal air mixing (shown by horizontal arrows in Fig. 16a).

Nevertheless, we shall discuss some common ideas and give estimations on ozone change inside the vortex in the winter-spring season taking into account charge particles effects on the ozone and some other compounds destruction.

In Fig. 16b the altitudinal distribution of the condensation nuclei is presented, which was typical for some of the ER-2 flights in the beginning of September 1987 as a part of the AAOE program [63]. The altitudinal distribution (1) corresponds to the condensation nuclei distribution inside the vortex, while the altitudinal distribution (2) corresponds to that one at the vortex circumference. According to our estimations the absolute rate of ozone depletion here can change in the range of $\sim(1.6-8.0) \times 10^{-7} \mathrm{~s}^{-1}$ (see Eq. (26)).

The analysis presented in Fig. 16b shows that inside the vortex the condensation nuclei distribution differs significantly from that one outside the vortex. An increased condensation nuclei concentration observed inside the vortex in the altitude range of $17-20 \mathrm{~km}$ because of the PSC presence, but outside the vortex there is a the monotonic increase of the condensation nuclei concentration above $\sim 14 \mathrm{~km}$. As one can see in Fig. $16 \mathrm{~b}$ the direct ozone destruction rate on the background ions in the absence of clouds at the altitudes of $\sim 14-18 \mathrm{~km}$ outside the vortex is higher than that one at the same altitudes inside the vortex. But that not at all means that ozone depletion forms outside the vortex.

First, together with the direct destruction of ozone, also decay the main chlorine reservoirs decay also and the $\mathrm{ClO}$ dimer cycle (and other halogen cycles) destruct ozone too. The cold temperatures at the centre of the vortex promote the accumulation of $\mathrm{ClO}$ as the result of sinking of air in the vortex to the levels with relatively high ion concentration. The cold temperatures also increase the efficiency of $\mathrm{ClO}$ dimer cycle in this region (19-24).

Second, we want to recall that the ozone hole formation is possible only since air inside the vortex is isolated relatively from the surrounding air masses, and the ozone content do not recover by the transfer of air masses from the zones with the enlarged ozone concentrations. That means that at the same ozone destruction rate by the background ions inside and outside the vortex, and the ozone concentrations in those zone can be strongly different from each other. Ozone can be almost completely depleted inside the vortex, and that is not possible for ozone outside the vortex, because for the latter case the equilibrium ozone concentrations will be determined not only by the photochemistry with the charged particles participation but by the atmospheric dynamics also.

That is why the comparatively slow but irretrievable ozone losses take place in inside vortex. The destructed ozone is replaced by the new portions of ozone moving slow from above. If the ozone and chlorine reservoirs decay rates are small, ozone accumulates, first of all in zones, where its decay rate is comparatively low, i.e. in the cloud and aerosol layers. While the Sun elevation angle rises the ozone destruction rate increases. The reason of that is not only the intensification of the halogen catalytic cycles and currents in the stratosphere (see above). While the temperature rises, the total PSC area decreases and the background ion contribution became stronger inside the vortex, especially at low altitudinal range $\sim 14-16$ $\mathrm{km}$. However, the further temperature growth with the solar elevation angle rise brings to the vortex destruction and ozone recovery. Besides that at a certain stage the so-called chlorine reservoir recovery 


\section{Polar vortex}

$\mathrm{O}_{3}, \mathrm{HCl}$, etc.

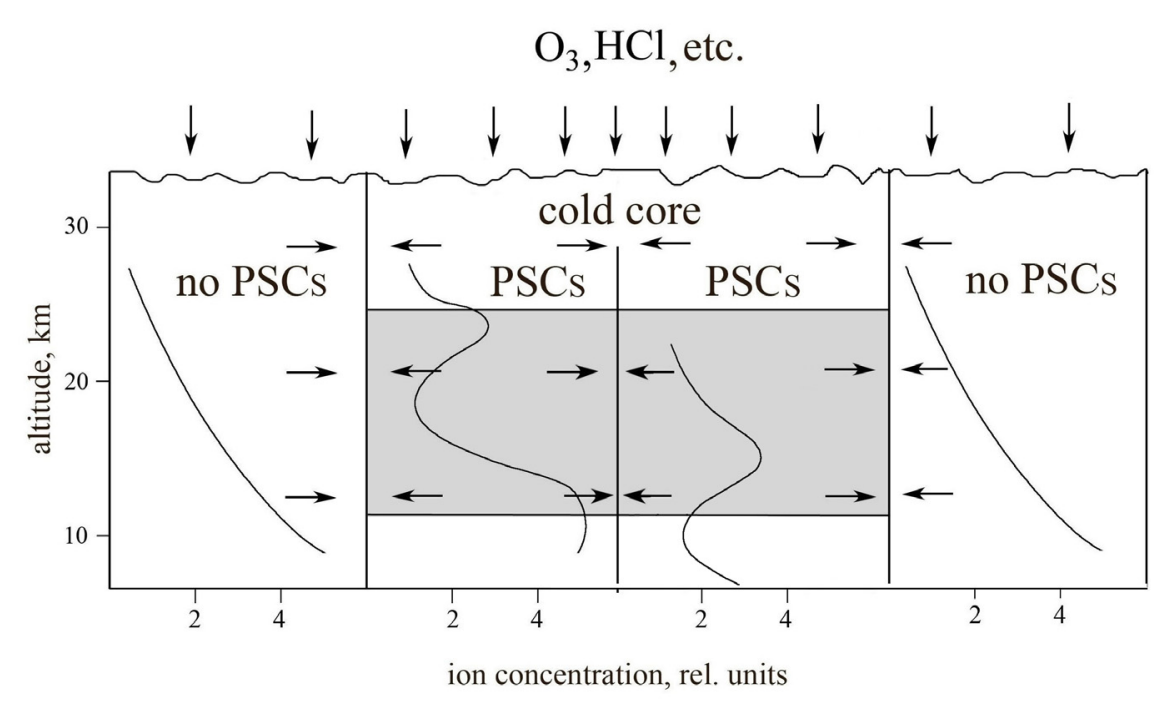

(a) (b)

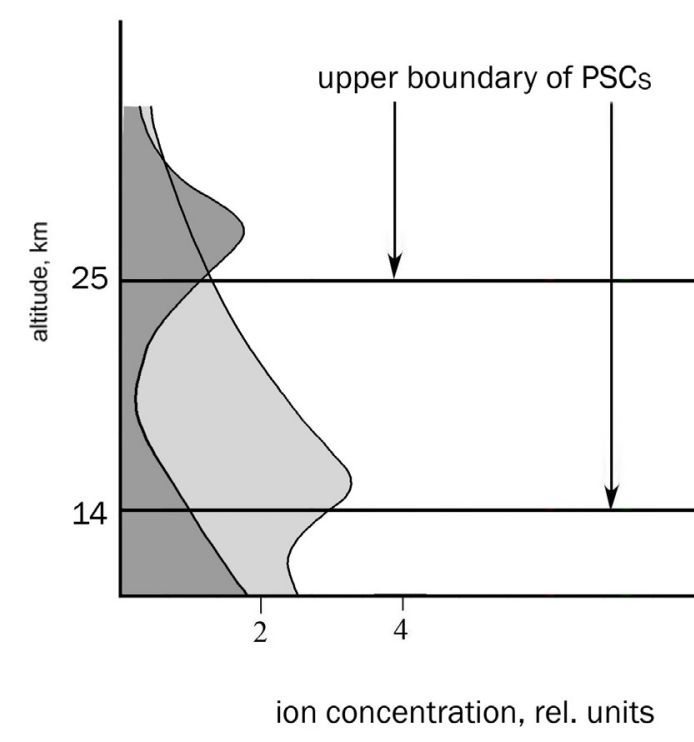

Fig. 16 The simplified scheme of the ozone depletion formation with the charged particles participation (see text). The altitude profiles of the ion concentration inside the cold vortex core, where PSCs are present at the different altitudes, and on the periphery of the vortex, free from clouds. The shaded area shows the altitudinal range of the PSC formation and their effect on the ionic concentration as a result of the global electric circuit work (a). Evaluations of the magnitude of the ion (CN) concentration and consequently the rate of destruction of the ozone at different altitudes at the circumference and inside the polar vortex according to the aircraft measurements as part of AAOE experiment [63] in the beginning of September 1987 (b). The altitude distributions of ions for different upper boundaries of PSCs (dark area for $25 \mathrm{~km}$ and light area for $14 \mathrm{~km})(\mathrm{c})$.

stage occurs, and the $\mathrm{ClO}$ dimer cycle, which also destruct ozone, stops to be active.

It should be pointed out also that the ozone and chlorine reservoirs decay rates inside the vortex substantially depend on the polar stratospheric clouds altitude (Fig. 16c). For example, in the case of the
PSC altitude of $\sim 14 \mathrm{~km}$, which is typical for the central zones of the Antarctic polar vortex, all ions above this level correspond to, or even exceed the background ions distribution (Fig. 16c). Besides that the ozone destruction in the middle zone of vortex at such altitude distribution of ions can be much more 
effective than at the vortex circumference, where air enriched by ozone transpires from the regions outside the vortex.

In Ref. [22] we showed that the ozone depletion development in Arctic at the altitudes about $\sim 16 \mathrm{~km}$ can significantly lag behind the maximum of the chlorine accumulation, and this confirms, in our opinion, the possibility of the direct destruction of ozone by the background ions in the polar vortex in the middle and end of spring.

As it was mentioned above one of the key processes, which account for the ozone loss inside the vortex, is the atmospheric dynamics, i.e. the vortex power. The PSCs are attributed to low temperature and well-developed atmospheric dynamics, as the more dynamics becomes stronger the more ozone volume descend at those altitudes, where enhanced content of the ions is observed (Figs. 5 and 16a).

The natural question appears in the connection to the non-halogen ozone destruction mechanism on the charged particles. What role it could play in 1980s when the growth of halogen production in the industrial epoch played the main role in the ozone holes formation processes. However the possible reason for the appearance of ozone holes in the early 1980s might not be solely because of the increase in chlorine species in the lower stratosphere but also because of a change in the atmospheric dynamics and temperature decrease in the lower stratosphere [70, 71]. As a result, ozone and the additional chlorine species descended to the altitude at which a higher concentration of ions prevailed and were destroyed there.

Thus, the cause of the ozone holes formation could be the combined factors, and their explanation and the forecast of those holes without an application of the non-halogen mechanism seems to be a difficult task, especially for Arctic regions. In our research study [22] a number of facts are collected which relate to the ozone holes formations which are difficult to explain solely from the position of the halogen mechanism.
Besides that the non-halogen mechanism, as it was mentioned above, can play a noticeable role in the mesosphere and the troposphere. Non-halogen mechanism is related to the global electric circuit. The current system embraces the whole atmosphere from the D-region of the ionosphere ( 60-90 km) to surface layers and possesses a complicated structure. The solar activity variations give rise to the current system change which causes the change in the ozone content in the atmosphere and solar radiation variations in the visible and UV spectral intervals. That can cause direct effects on our planet climate.

\section{Conclusion}

Thus, our analysis of published results of experiments in the Polar Regions and the theoretical estimates showed that alongside with the chlorine mechanism of the ozone destruction there is a mechanism of ozone decay on the charged particle, primarily on ions, which can act also at those latitudes and altitudes where chlorine oxide $\mathrm{ClO}$ is absent, as well as in night conditions.

This mechanism allows to explain the ozone depletion formation in the Polar Regions without applying chlorine mechanism. At the same time it does not mean that a chlorine mechanism does not work. The proportion of the each mechanism contribution should be defined further more exactly.

In the presence of the charged particles, together with ozone some other chemical compounds, including such stable molecules as $\mathrm{CH}_{4}$ and $\mathrm{N}_{2} \mathrm{O}$, as well as a number of the chlorine containing species such as $\mathrm{ClONO}_{2}, \mathrm{HCl}, \mathrm{CFCl}_{3}, \mathrm{CFCl}_{2}, \mathrm{CCl}_{4}$ and some other are undergone to the chemical decay. The degradation of the chlorine reservoirs of $\mathrm{HCl}$ and $\mathrm{ClONO}_{2}$ brings to formation of the active chlorine which can intensify the ozone destruction effect.

The proposed mechanism of the destruction of the above mentioned species including ozone, assumes the existence of the chemical reactions on the effective surface of the strongly charged particles with the use 
of the additional energy which molecule acquire. The main channel of the compounds destruction on the strongly charged particles surfaces is the interaction of molecules with hydroxyl $\mathrm{OH}$. The main supplier of $\mathrm{OH}$ to the charged particles surfaces is ozone, and at a lesser extent, $\mathrm{HOONO}$ and $\mathrm{HNO}_{4}$.

The analysis shows that among the charged particles the most favoured agent for the considered above processes is $\mathrm{H}_{3} \mathrm{O}^{+}\left(\mathrm{H}_{2} \mathrm{O}\right)_{\mathrm{n}}$ ion with the small number of ligands (presumably $\mathrm{n}<10$ ). It is those ions which are concentrated on upper boundaries of the polar stratospheric clouds and aerosol layers as a result of the global electric circuit work. We do not exclude thus a possible role of other charged particles, as negatively charged ions or the charged ice crystals in processes under consideration.

The distribution of stratospheric ions is determined by several factors. Primary ions, as shown in the article, can serve as condensation centres for liquids that form PSC particles and aerosol layers. Particularly effective is the formation of drops consisting of water with the addition of surfactants. Nitric acid might play this role in the polar stratosphere.

The processes of the drops formation on ions as well as the adherence of ions to aerosols and PSC particles reduce the concentration of ions inside the clouds and contribute to the accumulation of unipolar ions at the lower and upper boundaries of the polar stratospheric clouds and aerosol layers as a result of the work of the global electric circuit.

Results of the global electric circuit work (the accumulation of ions) expose itself not only at the upper and lower cloud boundaries but also at the every local level, thus enhancing the presence of ions of both signs in the interspaces of the aerosol and clouds layers. The "uneven" vertical profile of the clouds and aerosol layers well as their uneven spatial distribution gives rise to the "uneven" ozone and other compounds distribution as a result of their interaction with ions.

The global electric circuit functioning and consequently, the ion accumulation become stronger in the daytime, because the electric currents in the stratosphere expose an essential diurnal range [46].

The stratospheric dynamics, i.e., the sinking of the air in the vortex, is a key process in the formation of the ozone and other "holes". The flow of the air containing the species which would be disintegrated by ions descends from the heights with low ion concentration to the levels with relatively high ion concentration.

Our analysis of the polar experiments shows that concentrations of condensation nuclei (ions) in the Antarctic polar stratosphere in the winter and spring period exceeds significantly (can achieves an order of magnitude) the concentrations of condensation nuclei (ions) in the Arctic polar stratosphere for the similar period. In our opinion, that phenomenon together with atmospheric dynamics accounts for the formation of well-developed large ozone holes in Antarctic, while in Arctic mainly mini-holes are observed.

The considered mechanism of ozone and other compounds destruction on the charged particles can work also in the mesosphere and the troposphere.

The solar activity variations cause the electric currents variations in the whole atmosphere at the altitudes of $\sim 0-90 \mathrm{~km}$ and affect the total ozone content and radiation field change in the UV and visible spectral interval at the Earth surface. That can bring to direct influence on the climate of our planet.

Further experimental research on the distribution of ions and other charged particles in the polar stratosphere, the effects of PSCs and aerosol layers on this distribution, and the experimental confirmation (both in situ and in the laboratory) of the mechanism of photochemical reactions involving the participation of charged particles is of great importance.

\section{References}

[1] Brasseur, G., and Solomon, S. 1986. Aeronomy of the Middle Atmosphere. D. Reidel Publishing Company, 452.

[2] Kamsali, N., and Chandramma, S. 2011. "Ion Density in the Stratosphere." International Journal of Physics and 
Applications 3 (1): 117-23. ISSN 0974-3103. Available online: http://www.irphouse.com.

[3] Morita, Y., and Ishikawa, H. 1970. "On the Possible Role of Ozone Affecting the Atmospheric Electricity in the Stratosphere." J. Atmospheric and Terrestrial Physics 32: 1495-599.

[4] Lapshin, V. B., Yablokov, M. Y., and Palei, A. A. 2002. "Vapor Pressure over a Charged Drop." Russian J. Phys. Chem. 76: 1727-9.

[5] Nielsen, J. K., Maus, C., Rzesanke, D., and Leisner, T. 2011. "Charge Induced Stability of Water Droplets in Subsaturated Environment." Atmos. Chem. Phys. 11: 2031-7.

[6] Smirnov, V. M. 1975. "Ecological Problems of the Earth's Atmosphere.” Sov. Phys. Usp. 18: 804-14.

[7] Larin, I. K., and Talrose, V. L. 1977. "Conditions and Possible Scale of Influence of the Charged Particles on Destruction of Ozone in an Atmosphere." Proceedings of the USSR Academy of Sciences 233 (3): 410-3.

[8] Arnold, S. T., and Viggiano, A. A. 1997. "Effect of Ion Hydration in the Gas-Phase Proton-Transfer Reaction of OH- with HBr." J. Phys. Chem. A 101: 2859-61.

[9] Viggiano, A. A., Dale, F., and Paulson, J. F. 1988. "Proton Transfer Reactions of $\mathrm{H}+\left(\mathrm{H}_{2} \mathrm{O}\right) n=2-11$ with Methanol, Ammonia, Pyridine, Acetonitrile and Acetone." J. Chem. Phys. 88: 2469-77.

[10] Kawai, Y., Yamaguchi, S., Okada, Y., Takeuchi, K., Yamauchi, Y., Ozawa, Sh., and Nakai, H. 2003. "Reactions of Protonated Water Clusters $\mathrm{H}+\left(\mathrm{H}_{2} \mathrm{O}\right)$ n $(\mathrm{n}=1-6)$ with Dimethylsulfoxide in a Guided Ion Beam Apparatus." Chemical Physics Letters 377: 69-73.

[11] Lovejoy, E. R. 1999. "Ion Trap Studies of $\mathrm{H}+\left(\mathrm{H}_{2} \mathrm{SO}_{4}\right) \mathrm{m}\left(\mathrm{H}_{2} \mathrm{O}\right)$ n Reactions with Water, Ammonia, and a Variety of Organic Compounds." International Journal of Mass Spectrometry 190/191: 231-41.

[12] Wincel, H., Mereand, E., and Castleman, A. W. Jr. 1994. "Reactions of $\mathrm{N}_{2} \mathrm{O}_{5}$ with Protonated Water Clusters H+(H20)n , n = 3-30." J. Phys. Chem. 98: 8606-10.

[13] Varotsos, C. A., Cracknell, A. P., and Tzanis, C. 2010. "Major Atmospheric Events Monitored by Deep Underground Muon Data." Remote Sensing Letters 1 (3): 169-78.

[14] Yu, F. 2004. "Formation of Large NAT Particles and Denitrification in Polar Stratosphere: Possible Role of Cosmic Rays and Effect of Solar Activity." Atmos. Chem. Phys. 4: 2273-83.

[15] Lu, Q. B., and Sache, L. 2001. "Effect of Cosmic Rays on Atmospheric Chlorofluorocarbon and Ozone Depletion." Phys. Rev. Lett. 87: 078501.

[16] Lu, Q. B., and Madey, T. E. 2001. "Giant Enhancement of Electron-Induced Dissociation of Chlorofluorocarbons Coadsorbed with Water or Ammonia Ices: Implications for Atmospheric Ozone Depletion.” J. Chem. Phys. 111: 2861-4.

[17] Lu, Q. B. 2010. "Cosmic-Ray-Driven Electron-Induced Reactions of Halogenated Molecules Adsorbed on ice Surfaces: Implications for Atmospheric Ozone Depletion and Global Climate Change." Physics Reports 487: 141-67.

[18] Lu, Q. B. 2012. “On Cosmic-Ray-Driven Electron Reaction Mechanism for Ozone Hole and Chlorofluorocarbon Mechanism for Global Climate Change." arXiv: 1210.1498 [physics.ao-ph].

[19] Lu, Q. B. 2013. "Cosmic-Ray-Driven Reaction and Greenhouse Effect of Halogenated Molecules: Culprits for Atmospheric Ozone Depletion and Global Climate Change." Int. J. Mod. Phys. B 27: 1350073 (1-38).

[20] Lu, Q. B. 2015. New Theories and Predictions on the Ozone Hole and Climate Change. World Scientific Publishing Co.

[21] Belikov, Y. E., and Nikolayshvili, S. Sh. 2012. "Possible Mechanism of Ozone Destruction on Ice Crystals in the Polar Stratosphere." Russian Meteorology and Hydrology: 37 (10): 666-73.

[22] Belikov, Y. E., and Nikolayshvili, S. Sh. 2015. "Ozone Holes: New Approach." Earth and Universe (Russian Edition) 2: 27-39.

[23] Latif, M. T., Dominick, D., Ahamad, F., Khan, M. F., Juneng, L., and Hamzah, F. M. 2014. "Long Term Assessment of Air Quality from a Background Station on the Malaysian Peninsula." Science of the Total Environment 482: 336-48.

[24] Wei-Zhen, L., and Dong, W. 2008. "Ground-Level Ozone Prediction by Support Vector Machine Approach with a Cost-Sensitive Classification Scheme." Science of the Total Environment 395: 109-16.

[25] Savelyev, I. V. 1980. "Electric Field in a Vacuum." In Physics, a general course. Moscow: MIR Publisher. Volume 2, 11-60.

[26] Reist, P. C. 1984. Introduction to Aerosol Science. New York: Macmillan Publishing Company, 299.

[27] Chukin, V. V. 2005. Physical Properties of the Atmosphere. St. Petersburg, Russia: Publisher System, Russian Edition, 112.

[28] Barret, J. 2003. "Liquid Water and Hydration of Ions." Book Inorganic Chemistry in Aqueous Solution. The Royal Society of Chemistry, 184.

[29] Kammeyer, C. W., and Whitman, D. R. 1972. "Quantum Mechanical Calculation of Molecular Radii. I. Hydrides of Elements of Periodic Groups IV through VII." $J$. Chemical Physics 56 (9): 4419-21.

[30] Sander, S. P., Sander, S. P., Finlayson-Pitts, B. J., Friedl, R. R., Golden, D. M., Huie, R. E., Keller-Rudek, H., Kolb, C. E., Kurylo, M. J., Molina, M. J., Moortgat, G. K., 
Orkin, V. L., Ravishankara, A. R., and Wine, P. H. 2006. "Chemical Kinetics and Photochemical Data for Use in Atmospheric Studies." Tech. Rep. JPL Publ. 06-2: Jet Propulsion Laboratory, Evaluation number 15, Pasadena, USA.

[31] Intezarova, E. I., and Kondrat'ev, V. N. 1967. "Thermal Decomposition of Ozone.” Izv. Akad. Nauk SSSR. Ser. Khim. (Russian Edition) 11: 2440-6.

[32] von Hobe, M. et al. 2005. "A Re-evaluation of the $\mathrm{ClO} / \mathrm{Cl}_{2} \mathrm{O}_{2}$ Equilibrium Constant Based on Stratospheric in Situ Observations." Atmos. Chem. Phys. 5: 693-702.

[33] Shamir, N., and van Driel, H. M. 2000. "Trapping and Detrapping of Electrons Photo Injected from Silicon to Ultrathin $\mathrm{SiO}_{2}$ over Layers. II. In $\mathrm{He}, \mathrm{Ar}, \mathrm{H} 2, \mathrm{~N} 2, \mathrm{CO}$, and $\mathrm{N}_{2}$ O." J. Appl. Phys. 88: 909-17.

[34] Shamir, N., Mihaychuk, J. G. van Driel, H. M., and Kreuzer, H. J. 1999. "Universal Mechanism for Gas Adsorption and Electron Trapping on Oxidized Silicon." Phys. Rev. Lett. 82: 359-61.

[35] Hofmann, D. J. et al. 1989. "Stratospheric Clouds and Ozone Depletion in the Arctic during January 1989." Nature 340: 117-21.

[36] Hofmann, D. J. 1990. "Measurement of the Concentration Nuclei Profile to $31 \mathrm{~km}$ in the Arctic in January and Comparison with Antarctic Measurements." Geophys. Res. Lett. 17 (4): 357-60.

[37] Schlager, H., and Arnold, F. 1990. "Measurements of Stratospheric Gaseous Nitric Acid in the Winter Arctic Vortex Using a Novel Rocket Borne Mass Spectrometric Method." Geophys. Res. Lett. 17 (4): 433-6.

[38] Oppenheimer, M. 1987. "Stratospheric Sulphate Production and the Photochemistry of the Antarctic Circumpolar Vortex." Nature 328: 702-4.

[39] Zhao, J., Toon, O. B., and Turco, R. P. 1995. "Origin of Condensation Nuclei in the Springtime Polar Stratosphere.” J. Geophys. Res. 100 (D3): 5215-27.

[40] Renard, J. B., Tripathi, S. N., Michael, M. et al. 2013. "In Situ Detection of Electrifies Aerosols in the Upper Troposphere and Stratosphere." Atmos. Chem. Phys. 13: $1-8$.

[41] Hofmann, D. J., and Rosen, J. M. 1985. "Antarctic Observation of Stratospheric Aerosol and High Altitude Condensation Nuclei Following the El Chichon Eruption." Geophys. Res. Lett. 17 (12): 13-6.

[42] Bering, E. A. 1995. "The Global Circuit: Global Thermometer, Weather By-product or Climatic Modulator.” Rev. Geophys. Supplement: 845-62.

[43] Williams, E. R. 2009. "The Global Electrical Circuit: A Review." Atmospheric Research 91: 140-52.

[44] Mareev, E. A. 2010. "Global Electric Circuit Research: Achievements and Prospects." Phys. Usp. 53: 504-11.

[45] Harrison, R. G., and Carslaw, K. S. 2003.
"Ion-Aerosol-Cloud Processes in the Lower Atmosphere." Review of Geophysics 41 (3): 1012.

[46] Holzworth, R. H., Bering, E. A. III, Kokorowski, M. F., et al. 2005. "Balloon Observations of Temporal Variation in the Global Circuit Compared to Global Lightning Activity." Advances in Space Research 36: 2223-8.

[47] Srivastava, A. K., and Tripathi, S. N. 2010. "Numerical Study for Production of Space Charge within the Stratiform Cloud.” J. Earth Syst. Sci. 119 (5): 627-38.

[48] Nicoll, K. A., and Harrison, R. G. 2010. "Experimental Determination of Layer Cloud Edge Charging from Cosmic Ray Ionisation." Geophysical Research Letters 37: L13802.

[49] Hoppel, W. A., and Frick, G. M. 1986. "Ion-Aerosol Attachment Coefficients and the Steady-State Charge on Aerosols in a Bipolar Ion Environment." Aerosol Sci. Tech. 5: 1-21.

[50] Griffiths, R. F., Latham, J., and Myers, V. 1974. "The Ionic Conductivity of Electrified Clouds.” Quart. J. Roy. Meteor. Soc. 100: 181-90.

[51] Chiu, C. S. 1978. "Numerical Study of Cloud Electrification in an Axisymmetric, Time-Dependent Cloud Model." J. Geophys. Res. 83: 5025-49.

[52] Kamm, S., Mohler, O., Naumann, K. H., Saathoff, H., and Schurath, U. 1999. "The Heterogeneous Reaction of Ozone with Soot Aerosol." Atmospheric Environment 33: 4651-61.

[53] Holzworth, R. H. 1991. "Conductivity and Electric Field Variations with Altitude in the Stratosphere." J. Geophys. Res. 96 (D7): 12857-64.

[54] World Meteorological Organization (WMO). 1999. "Scientific Assessment of Ozone Depletion." Global Ozone Res. and Monit. Proj., Geneva, Rep. 44.

[55] Wilson, C. 1912. "On an Expansion Apparatus for Making Visible the Tracks of Ionising Particles in Gases and Some Results Obtaining by Its Use." Proc. Roy. Soc. London A 87: 277.

[56] Nadykto, A. B., Makela, J. M., Fangqun, Yu, Kulmala, M., and Laaksonen, A. 2003. "Comparison of the Experimental Mobility Equivalent Diameter for Small Cluster Ions with Theoretical Particle Diameter Corrected by Effect of Vapour Polarity." Chemical Physics Letters 382: 6-11.

[57] Abramzon, A. A. 1981. Surface-Active Substances: Properties and Application. Russian edition 2, Chemistry: USSR, Leningrad, 304.

[58] D'Auria, R., and Turco, R. P. 2001. "Ionic Clusters in the Polar Winter Stratosphere.” Geophys. Res. Lett. 28 (20): 3871-4.

[59] Gumbel, J., and Witt G. 2002. "Cluster Ions and Ice Particle Nucleation: Positive Feedback at the Summer Mesopause." Geophys. Res. Lett. 29 (16): 29-1-29-4. 
[60] Stein, B., Immler, F., Mielke, B., Rairoux, P., Wedekind, C., and Woste L. 1996. "Characterization of Liquid and Solid PSC's by Multispectral Lidar.” XVIII Quadrennial Ozone Symposium, L'Aquila, Italy, 12-21 September, 611-4.

[61] Harrison, R. G., and Alpin, K. L. 2001. "Atmospheric Condensation Nuclei Formation and High-Energy Radiation." J. Atmospheric and Solar-Terrestrial Physics 63: 1811-9.

[62] SAGE III Ozone Loss and Validation Experiment 1999-2000 (SOLVE). Available online: http:/espoarchive.nasa.gov/archive/arcs/solve/data/.

[63] Airborne Antarctic Ozone Experiment 1987 (AAOE-87). Available online: http://badc.nerc.ac.uk/.

[64] Proffitt, M. H. et al. 1989. "Evidence for Diabatic Cooling and Poleward Transport within and around the 1987 Antarctic Ozone Hole.” J. Geophys. Res. 94 (D14): 16797-813.

[65] Anderson, J. G., Brune, W. H., and Toohey D. W. 1991. "Free Radicals within the Antarctic Vortex: The Role of CFCs in Antarctic Ozone Loss." Science 251: 39-46.

[66] NIST Chemical Kinetics Database: Standard Reference
Database 17, Version 7.0 (Web Version), Release 1.6.7, Data Version 2013.03. http://kinetics.nist.gov/.

[67] Fry, J. L., Nizkorodov, S. A., Okumura, M., Roehl, C. M., Francisco, J. S., and Wennberg, P. O. 2004. "Cis-cis and Trans-Perp HOONO: Action Spectroscopy and Isomerization Kinetics." J. Chem. Phys. 121 (3): 1432-48.

[68] Osborn, M. T., Pitts, M. S., Powell, K. A., and McCormick, M. P. 1990. "SAM II Aerosol Mesurements during the 1989 AASE." Geophysical Research Letters 17 (4): 397-400.

[69] Kent, G. S. et al. 1985. "Variation in the Stratospheric Aerosol Associated with the North Cyclonic Polar Vortexas Measured by the SAM II Satellite Sensor." J. Atmos. Sci. 42: 1536-51.

[70] Jadin, E. A. 2011. 'Stratospheric 'Wave Hole' and Interannual Variations of the Stratospheric Circulation in Late Winter." Natural Science 3 (4): 259-67.

[71] Jadin, E. A., Wei, K., Zyulyaeva, Yu. A., Chen, W., and Wang, L. 2010. "Stratospheric Wave Activity and the Pacific Decadal Oscillation." J. Atmospheric and Solar-Terrestrial Physics 72: 1163-70. 